\title{
Convolutions on compact groups and Fourier algebras of coset spaces
}

\author{
by \\ Brian E. Forrest (Waterloo), Ebrahim Samei (Saskatoon) \\ and Nico Spronk (Waterloo)
}

\begin{abstract}
We study two related questions. (1) For a compact group $G$, what are the ranges of the convolution maps on $\mathrm{A}(G \times G)$ given for $u, v$ in $\mathrm{A}(G)$ by $u \times v \mapsto u * \check{v}$ $\left(\check{v}(s)=v\left(s^{-1}\right)\right)$ and $u \times v \mapsto u * v$ ? (2) For a locally compact group $G$ and a compact subgroup $K$, what are the amenability properties of the Fourier algebra of the coset space $\mathrm{A}(G / K)$ ? The algebra $\mathrm{A}(G / K)$ was defined and studied by the first named author.

In answering the first question, we obtain, for compact groups which do not admit an abelian subgroup of finite index, some new subalgebras of $\mathrm{A}(G)$. Using those algebras we can find many instances in which $\mathrm{A}(G / K)$ fails the most rudimentary amenability property: operator weak amenability. However, using different techniques, we show that if the connected component of the identity of $G$ is abelian, then $\mathrm{A}(G / K)$ always satisfies the stronger property that it is hyper-Tauberian, which is a concept developed by the second named author. We also establish a criterion which characterises operator amenability of $\mathrm{A}(G / K)$ for a class of groups which includes the maximally almost periodic groups. Underlying our calculations are some refined techniques for studying spectral synthesis properties of sets for Fourier algebras. We even find new sets of synthesis and nonsynthesis for Fourier algebras of some classes of groups.
\end{abstract}

In a recent article [30, §3.3], the third named author posed a question which, for a compact group $G$, reduces to asking if the maps from $\mathrm{A}(G \times G)$ to $\mathrm{A}(G)$, given on elementary functions by $u \times v \mapsto u * \check{v}$ and $u \times v \mapsto$ $u * v$, are surjective. We show in Sections 2 and 4 that this is not the case when $G$ does not admit an abelian subgroup of finite index. Moreover, the ranges of both maps are quite different: the first gives us a new algebra $\mathrm{A}_{\Delta}(G)$, and the second gives us an algebra $\mathrm{A}_{\gamma}(G)$, which was originally discovered by B. E. Johnson [17]. It is worth noting that Johnson used $\mathrm{A}_{\gamma}(G)$ in a very clever way to show that for compact groups $G, \mathrm{~A}(G)$ is generally not amenable, in fact not even weakly amenable. Johnson's results were surprising when his article was published, since at the time the expectation

2010 Mathematics Subject Classification: Primary 43A30, 43A77, 43A85; Secondary 47L25.

Key words and phrases: convolution, coset space, Fourier algebra. 
was that amenability of $\mathrm{A}(G)$ would be equivalent to classical amenability for the underlying group $G$. Recent applications of the theory of operator spaces to the study of $\mathrm{A}(G)$ have given us a much better understanding of why $\mathrm{A}(G)$ fails to be amenable as a Banach algebra for any group which does not contain abelian subgroups of finite index. However, it is one of the main themes of this paper that subalgebras of $\mathrm{A}(G)$ such as $\mathrm{A}_{\gamma}(G)$ can shed further light on the amenability problem and allow us to deduce much more about the relationship between $\mathrm{A}(G)$ and $G$.

Motivated by Johnson's beautiful theorem that the group algebra $\mathrm{L}^{1}(G)$ of a locally compact group $G$ is amenable as a Banach algebra if and only if $G$ is amenable, together with the spectacular failure of the natural analog of this result for $\mathrm{A}(G)$, Z.-J. Ruan demonstrated the tremendous value in recognising the operator space structure on $\mathrm{A}(G)$ by introducing the concept of operator amenability and then using this to show that $\mathrm{A}(G)$ is operator amenable if and only if $G$ is amenable [25]. This was followed by the third named author [29], and independently the second named author [27], each establishing that $\mathrm{A}(G)$ is always operator weakly amenable. The general question of when $\mathrm{A}(G)$ is weakly amenable as a Banach algebra remains open. However, using, in part, techniques developed in the present article, the authors have recently shown that $\mathrm{A}(G)$ fails to be weakly amenable for any $G$ which contains a connected nonabelian compact subgroup. See [9].

For a compact subgroup $K$ of $G$, the Fourier algebra of the coset space, $\mathrm{A}(G / K)$, was described by the first named author [6]. A $(G / K)$ may be simultaneously viewed as an algebra of continuous functions on the coset space $G / K$ and as a sublgebra of $\mathrm{A}(G)$. The latter view allows us to define a natural operator space structure on $\mathrm{A}(G / K)$. It was shown in [6] that many properties of $\mathrm{A}(G / K)$ associated with amenability, such as existence of a bounded approximate identity, and factorisation, are closely linked to such properties of $\mathrm{A}(G)$. Thus we are naturally led to consider the amenability properties of $\mathrm{A}(G / K)$. Surprisingly, positive results are rather sparse, even in the category of operator spaces. In Section 3 we establish that whenever $G$ has a compact connected nonabelian subgroup $K$, then there exists a compact subgroup $K^{*}$ of $G \times G$ such that $\mathrm{A}\left(G \times G / K^{*}\right)$ is not operator weakly amenable. This contrasts sharply with the positive result of [29, 27], mentioned above. As a complement, we establish in Section 3.3 that $\mathrm{A}(G / K)$ is hyper-Tauberian whenever the connected component of the identity $G_{e}$ in $G$ is abelian. The hyper-Tauberian property, developed by the second named author [28], implies weak amenability of a commutative semisimple Banach algebra. Note that this result does not address the operator space structure of $\mathrm{A}(G)$ at all. This generalises a result of the first named author and V. Runde [8] that $\mathrm{A}(G)$ is weakly amenable when $G_{e}$ is abelian. In Section 3.4 we obtain, for certain groups which we call $[\mathrm{MAP}]_{K}$-groups, a characterisation of when 
$\mathrm{A}(G / K)$ is operator amenable. Using related techniques, we also find new sets of local synthesis for $\mathrm{A}(G \times G)$ when $G$ has an abelian connected component of the identity, ones which are not known to be in the closed coset ring of $G \times G$.

Many of the results of Section 3 rely heavily on constructions relating to convolutions on compact groups. In Section 1 we develop a general framework in which to view the "twisted" convolution $f \times g \mapsto f * \check{g}$ on functions on compact $G$. We realise the image of this map as a special example of functions on a coset space. We find that our general framework naturally accommodates an easy, though far reaching generalisation of a result relating spectral information between various different algebras, obtained for abelian compact groups by N. Th. Varapolous [33], and generalised to arbitrary compact groups by L. Turowska and the third named author; see Theorem 1.4. Since it takes little extra effort, we prove our spectral results for a class we call $[\mathrm{MAP}]_{K}$-groups, which includes maximally almost periodic groups. In Section 2 we apply our twisted convolution framework to $\mathrm{A}(G \times G)$. In doing so, we obtain not one, but an infinite sequence of new subalgebras of $\mathrm{A}(G)$, when $G$ does not admit an abelian subgroup of finite index. In Section 4 we provide a framework for convolutions on compact $G$, and show how it differs from the twisted convolution when applied to $\mathrm{A}(G \times G)$. In effect, we have an alternative method to obtain the algebra $\mathrm{A}_{\gamma}(G)$ of Johnson.

As well as answering questions posed by the third author in [30], many of the ideas and techniques of the present article have inspired new work of the authors. In [9] the present authors have obtained a general weak amenability characterisation for $\mathrm{A}(G)$ when $G$ is compact. Moreover, general amenability properties of algebras $\mathrm{A}_{\Delta}(G)$ have been characterised, improving on the present article's Theorem 3.1, and partially generalising Theorem 3.9. In [23], D. Park and the second author have studied weak synthesis for sets which occur in Corollary 3.2 amongst other related sets.

0.1. Background and notation. The Fourier algebra $\mathrm{A}(G)$ of a locally compact group $G$ was defined by Eymard [5]. For compact $G$, there is an alternative description in [14, Chap. 34]. That the two descriptions coincide can be seen by comparing [5, p. 218] with [14, (34.16)]. We note that the Fourier algebra is closed under both group actions of left and right translations $(s, u) \mapsto s * u, s \cdot u: G \times \mathrm{A}(G) \rightarrow \mathrm{A}(G)$, given by

$$
s * u(t)=u\left(s^{-1} t\right), \quad s \cdot u(t)=u(t s) .
$$

Moreover, these actions are continuous in $G$ and isometric on $\mathrm{A}(G)$. We note that $\mathrm{A}(G)$ admits a von Neumann algebra $\mathrm{VN}(G)$ as its dual space. As such, it comes equipped with a natural operator space structure. See [4], for 
example, for more on this. We use the same definitions as [4] for completely bounded map, complete isometry and complete quotient map. We note that right and left translations on $\mathrm{A}(G)$, being the preadjoints of multiplications by unitaries on $\operatorname{VN}(G)$, are complete isometries.

Our main references for amenability are [16] and [26]. A Banach algebra $\mathcal{A}$ is said to be amenable if for any Banach $\mathcal{A}$-bimodule $\mathcal{X}$, and any bounded derivation $D: \mathcal{A} \rightarrow \mathcal{X}^{*}$, where $\mathcal{X}^{*}$ is the dual space with adjoint module actions, $D$ is inner. For a commutative Banach algebra $\mathcal{A}$, we say $\mathcal{A}$ is weakly amenable if for any symmetric $\mathcal{A}$-bimodule $\mathcal{X}$, the only bounded derivation $D: \mathcal{A} \rightarrow \mathcal{X}$ is 0 ; this is equivalent to having the same happen for $\mathcal{X}=\mathcal{A}^{*}$. Weak amenability for commutative Banach algebras was introduced in [1]. For both amenability and weak amenability there are some homological characterisations; see [18, 2, 12, 26].

Operator space notions of amenability and weak amenability were introduced in [25] and in [10] respectively, specifically for use with $\mathrm{A}(G)$. If $\mathcal{A}$ is a commutative Banach algebra which is also an operator space we say $\mathcal{A}$ is a completely contractive Banach algebra if the multiplication map $\mathcal{A} \times \mathcal{A} \rightarrow \mathcal{A}$ is completely contractive in the sense of [4, Chap. 7]. An operator space $\mathcal{V}$ is a completely contractive $\mathcal{A}$-module if it is an $\mathcal{A}$-module for which the module maps $\mathcal{A} \times \mathcal{V}, \mathcal{V} \times \mathcal{A} \rightarrow \mathcal{V}$ are completely contractive. The class of completely contractive $\mathcal{A}$-modules is closed under taking dual spaces with adjoint actions. We say $\mathcal{A}$ is operator (weakly) amenable if every completely bounded derivation $D: \mathcal{A} \rightarrow \mathcal{V}^{*}$ (with $\mathcal{V}=\mathcal{A}$ ) is inner (zero). Many of the homological characterisations alluded to above carry over to this setting, though with Banach space projective tensor products $\otimes^{\gamma}$ replaced by operator space projective tensor products $\widehat{\otimes}$.

Let $\mathcal{A}$ be a commutative semisimple (completely contractive) Banach algebra. Suppose $\mathcal{A}$ is regular on its spectrum $X=\Sigma_{\mathcal{A}}$; we regard $\mathcal{A}$ as an algebra of functions on $X$. If $\varphi \in \mathcal{A}^{*}$ we define

$$
\operatorname{supp}(\varphi)=\left\{x \in X: \begin{array}{ll}
\text { for every neighbourhood } U \text { of } x \text { there is } f \\
\text { in } \mathcal{A} \text { such that } \operatorname{supp}(f) \subset U \text { and } \varphi(f) \neq 0
\end{array}\right\} .
$$

Here $\operatorname{supp}(f)=\overline{\{x \in X: f(x) \neq 0\}}$. An operator $T: \mathcal{A} \rightarrow \mathcal{A}^{*}$ is called a local map if

$$
\operatorname{supp}(T f) \subset \operatorname{supp}(f)
$$

for every $f$ in $\mathcal{A}$. We say $\mathcal{A}$ is (operator) hyper-Tauberian if every (completely bounded) bounded local map $T: \mathcal{A} \rightarrow \mathcal{A}^{*}$ is an $\mathcal{A}$-module map. This concept was developed in [28] to study the reflexivity of the (completely bounded) derivation space of $\mathcal{A}$, and it generalises (operator) weak amenability.

For a commutative semisimple Banach algebra which is regular on its spectrum we have the following implications: 


$$
\text { amenable } \Rightarrow \text { weakly amenable } \Leftarrow \text { hyper-Tauberian }
$$

Moreover, if $\mathcal{A}$ is a completely contractive Banach algebra, each property implies its operator analogue, and the operator analogues satisfy the same implications.

\section{Algebras on coset spaces and a twisted convolution on com- pact groups}

1.1. The basic construction. Let $G$ be a locally compact group. Let $\mathcal{A}(G)$ be a Banach algebra of continuous functions on $G$ which is closed under right translations and such that for any $f$ in $\mathcal{A}(G)$ we have

- $\|s \cdot f\|=\|f\|$ for any $s$ in $G$,

- $s \mapsto s \cdot f$ is continuous.

If, moreover, $\mathcal{A}(G)$ is an operator space, we require for each $t$ in $G$ that $f \mapsto t \cdot f$ is a complete isometry.

If $K$ is a compact subgroup of $G$ we let

$$
\mathcal{A}(G: K)=\{f \in \mathcal{A}(G): k \cdot f=f \text { for each } k \text { in } K\},
$$

which is a closed subalgebra of $\mathcal{A}(G)$ whose elements are constant on left cosets of $K$. We let $G / K$ denote the space of left cosets with the quotient topology. We define two maps

$$
P: \mathcal{A}(G) \rightarrow \mathcal{A}(G), \quad P f=\int_{K} k \cdot f d k,
$$

and

$$
M: \mathcal{A}(G: K) \rightarrow \mathcal{C}_{b}(G / K), \quad M f(s K)=f(s) .
$$

The map $P$ is to be regarded as a Bochner integral over the normalised Haar measure on $K$; its range is $\mathcal{A}(G: K)$ and $P$ is a (completely) contractive projection. The map $M$ is well-defined by comments above, and its range consists of continuous functions since $\mathcal{A}(G: K) \subset \mathcal{C}_{b}(G: K)$. We note that $M$ is an injective homomorphism and denote its range by $\mathcal{A}(G / K)$. We assign a norm (operator space structure) to $\mathcal{A}(G / K)$ in such a way that $M$ is a (complete) isometry. We finally define two maps

$$
N=M^{-1}: \mathcal{A}(G / K) \rightarrow \mathcal{A}(G) \quad \text { and } \quad \Gamma=M \circ P: \mathcal{A}(G) \rightarrow \mathcal{A}(G / K)
$$

so $N$ is a (completely) isometric homomorphism and $\Gamma$ is a (complete) quotient map.

Let us record some basic properties of $\mathcal{A}(G / K)$. For a commutative Banach algebra $\mathcal{A}$, we let $\Sigma_{\mathcal{A}}$ denote its Gelfand spectrum.

PROPOSITION 1.1.

(i) Suppose $\mathcal{A}(G)$ is regular on $G$ and $G$ separates the points of $\mathcal{A}(G)$. Then $\mathcal{A}(G / K)$ is regular on $G / K$ and $G / K$ separates the points 
of $\mathcal{A}(G / K)$. Moreover, if $\Sigma_{\mathcal{A}(G)} \cong G$ via evaluation maps, and $K$ is a set of spectral synthesis for $\mathcal{A}(G)$, then $\Sigma_{\mathcal{A}(G / K)} \cong G / K$ via evaluation maps.

(ii) If the subalgebra $\mathcal{A}_{c}(G)$ of compactly supported elements of $\mathcal{A}(G)$ is dense in $\mathcal{A}(G)$, then the algebra $\mathcal{A}_{c}(G / K)$ of compactly supported elements in $\mathcal{A}(G / K)$ is dense in $\mathcal{A}(G / K)$.

We remark that (i) applies to the Fourier algebra $\mathrm{A}(G)$ for any locally compact group $G$ and compact subgroup $K$ by [13] or [32].

Proof. (i) Same as [6, Thm. 4.1].

(ii) It is obvious that $\Gamma \mathcal{A}_{c}(G)=\mathcal{A}_{c}(G / K)$. If $w$ in $\mathcal{A}(G)$ is the limit of a sequence $\left(w_{n}\right) \subset \mathcal{A}_{c}(G)$, then $\left\|\Gamma w-\Gamma w_{n}\right\| \leq\left\|w-w_{n}\right\| \rightarrow 0$ as $n \rightarrow \infty$.

For Fourier algebras, we have the important identification

$$
\mathrm{A}(G) \widehat{\otimes} \mathrm{A}(H) \cong \mathrm{A}(G \times H)
$$

via $f \otimes g \mapsto f \times g$, where $\widehat{\otimes}$ denotes the operator projective tensor product. See [3]. This is known to fail when the usual projective tensor product $\otimes^{\gamma}$ is used [19].

Proposition 1.2. If $G$ and $H$ are locally compact groups with respective compact subgroups $K$ and $L$, then there is a complete isometry identifying

$$
\mathrm{A}(G / K) \widehat{\otimes} \mathrm{A}(H / L) \cong \mathrm{A}(G \times H / K \times L)
$$

given on elementary tensors by $f \otimes g \mapsto f \times g$.

Note that there is a natural homeomorphism $G \times H / K \times L \cong G / K \times H / L$, which is in fact a $G \times H$-space morphism.

Proof. We identify $\mathrm{A}(G / K) \cong \mathrm{A}(G: K)$, etc. We have the following commuting diagram:

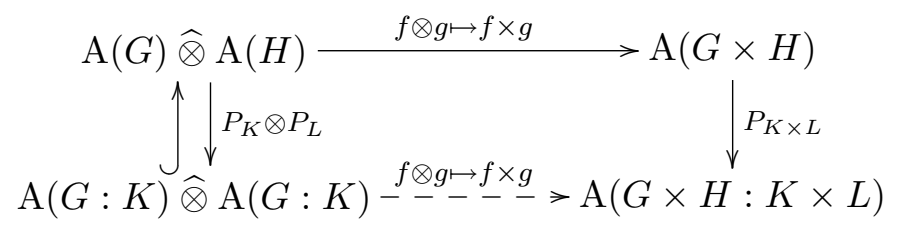

where the inclusion map $\mathrm{A}(G: K) \widehat{\otimes} \mathrm{A}(G: K) \hookrightarrow \mathrm{A}(G) \widehat{\otimes} \mathrm{A}(H)$ is a complete isometry, since each of $P_{K}$ and $P_{L}$ is a complete quotient projection. Notice that this inclusion map is a right inverse to $P_{K} \otimes P_{L}$. The desired map from $\mathrm{A}(G: K) \widehat{\otimes} \mathrm{A}(G: K)$ to $\mathrm{A}(G \times H: K \times L)$ completes this diagram, and may be realised as the composition of the inclusion map (up arrow) with the identification map (top arrow), and then $P_{K \times L}$. It is clear that $\left.P_{K \times L}\right|_{\overline{\operatorname{span}} \mathrm{A}(G: K) \times \mathrm{A}(H: L)}=\mathrm{id}$. Hence the desired map is injective. Since 
the map identifying $\mathrm{A}(G) \widehat{\otimes} \mathrm{A}(H) \cong \mathrm{A}(G \times H)$ is a complete isometry, and $P_{K} \otimes P_{L}$ and $P_{K \times L}$ are complete quotient maps, our desired map is a complete quotient map. Hence we have an injective complete quotient map, i.e. a complete isometry.

1.2. A twisted convolution. Let $G$ be a compact group for the remainder of this section. We use $G \times G$ in place of $G$ above, and $K=\Delta=$ $\{(s, s): s \in G\}$. Since the map

$$
(s, e) \Delta \mapsto s:(G \times G) / \Delta \rightarrow G
$$

is a homeomorphism, we identify the coset space with $G$. We observe that in this case the map $P: \mathcal{A}(G \times G) \rightarrow \mathcal{A}(G \times G)$ satisfies

$$
P w(s, t)=\int_{G} w(s r, t r) d r=\int_{G} w\left(s t^{-1} r, r\right) d r
$$

and the maps $M: \mathcal{A}(G \times G: \Delta) \rightarrow \mathcal{C}(G)$ and $N: \mathcal{C}(G) \rightarrow \mathcal{C}(G \times G: \Delta)$ satisfy

$$
M w(s)=w(s, e) \quad \text { and } \quad N f(s, t)=f\left(s t^{-1}\right) .
$$

We denote the range of $M$ by $\mathcal{A}_{\Delta}(G)$, and then $\left.N\right|_{\mathrm{A}_{\Delta}(G)}=M^{-1}$. The map $\Gamma=M \circ P$ above can be regarded as a "twisted" convolution, for if $\mathcal{A}(G \times G)$ contains an elementary function $f \times g$, then for $s$ in $G$,

$$
\Gamma(f \times g)(s)=\int_{G} f \times g(s t, t) d t=\int_{G} f(s t) g(t) d t=f * \check{g}(s) .
$$

We list some examples of $\mathcal{A}(G \times G)$ and $\mathcal{A}_{\Delta}(G)$.

(i) If $\mathcal{A}(G \times G)=\mathcal{C}(G \times G)$, then $\mathcal{C}_{\Delta}(G)=\mathcal{C}(G)$, by easy computation.

(ii) Let $\mathrm{V}(G \times G)=\mathcal{C}(G) \otimes^{h} \mathcal{C}(G)$ (Haagerup tensor product). Then $\mathrm{V}_{\Delta}(G)=\mathrm{A}(G)$, completely isometrically. See [31]. Note that by Grothendieck's inequality $\mathrm{V}(G \times G)=\mathcal{C}(G) \otimes^{\gamma} \mathcal{C}(G)$ (projective tensor product) isomorphically, though not isometrically.

(iii) Let $\mathcal{A}(G \times G)=\mathrm{A}(G) \otimes^{\gamma} \mathrm{A}(G)$. Then $\mathcal{A}_{\Delta}(G)=\mathrm{A}_{\gamma}(G)$ is the subalgebra of $\mathrm{A}(G)$ considered by B. Johnson [17]. He used it to study the amenability of $\mathrm{A}(G)$.

(iv) Consider $\mathrm{A}(G \times G) \cong \mathrm{A}(G) \widehat{\otimes} \mathrm{A}(G)$ (operator projective tensor product). The algebra $\mathrm{A}_{\Delta}(G)$ thus formed will be an essential object of our study.

We summarise some basic properties of the algebras $\mathcal{A}_{\Delta}(G)$ which clearly apply to the examples above. We say a norm (operator space structure) $\alpha$ on $\mathcal{X} \otimes \mathcal{Y}$ is (operator) homogeneous if for every pair of (completely) contractive linear maps $S: \mathcal{X} \rightarrow \mathcal{X}^{\prime}, T: \mathcal{Y} \rightarrow \mathcal{Y}^{\prime}$ the map $S \otimes T$ extends to a (complete) contraction from $\mathcal{X} \otimes^{\alpha} \mathcal{Y}$ to $\mathcal{X}^{\prime} \otimes^{\alpha} \mathcal{Y}^{\prime}$. 
Proposition 1.3.

(i) If there is a (completely contractive) Banach algebra $\mathcal{B}(G)$ of continuous functions on $G$ and a homogeneous (operator) norm (operator space structure) $\alpha$ on $\mathcal{B}(G) \otimes \mathcal{B}(G)$ so $\mathcal{A}(G \times G)=\mathcal{B}(G) \otimes^{\alpha} \mathcal{B}(G)$, then $\mathcal{A}_{\Delta}(G)$ is a (completely contractive) subalgebra of $\mathcal{B}(G)$.

(ii) If $\mathcal{A}(G \times G)$ is closed under left translations and the translation maps are continuous in $G \times G$ and (completely) isometric on $\mathcal{A}(G \times G)$, then $\mathcal{A}_{\Delta}(G)$ is closed under both left and right translations, and the translations are continuous on $G$, and (completely) isometric on $\mathcal{A}_{\Delta}(G)$.

Proof. (i) The map $M: \mathcal{A}(G \times G: \Delta) \rightarrow \mathcal{A}_{\Delta}(G)$ is the restriction of the slice map id $\otimes \delta_{e}: \mathcal{A}(G) \otimes^{\alpha} \mathcal{A}(G) \rightarrow \mathcal{A}(G)$ where $\delta_{e}$ is the evaluation functional at $e$.

(ii) For $f \in \mathcal{A}_{\Delta}(G), r$ in $G$ and any $(s, t) \in G \times G$ we have

$$
N(r \cdot f)(s, t)=f\left(s t^{-1} r\right)=f\left(s\left(r^{-1} t\right)^{-1}\right)=(e, r) * N f .
$$

Our assumptions ensure that the space $\mathcal{A}(G \times G: \Delta)$ is closed under left translations and each translation map is a (complete) isometry. For left translations we note that, for $r$ in $G$ and $f \in \mathrm{A}_{\Delta}(G)$, we have

$$
N(r * f)=(r, e) * N f
$$

and we argue as above.

1.3. Relationships between ideals. Let $\mathcal{A}$ be a Banach algebra contained in $\mathcal{C}_{0}(X)$ for some locally compact Hausdorff space $X$. For any closed subset $E$ of $X$ we define

$$
\begin{aligned}
& \mathrm{I}_{\mathcal{A}}(E)=\{f \in \mathcal{A}: f(x)=0 \text { for } x \in E\}, \\
& \mathrm{I}_{\mathcal{A}}^{0}(E)=\{f \in \mathcal{A}: \operatorname{supp}(f) \cap E=\emptyset \text { and } \operatorname{supp}(f) \text { is compact }\}, \\
& \mathrm{J}_{\mathcal{A}}^{0}(E)=\left\{f \in \mathrm{I}_{\mathcal{A}}(E): \operatorname{supp}(f) \text { is compact }\right\},
\end{aligned}
$$

where $\operatorname{supp}(f)=\overline{\{x \in X: f(x) \neq 0\}}$. If $X=\Sigma_{\mathcal{A}}$ and $\mathcal{A}$ is regular on $X$, we say $E$ is a set of spectral synthesis for $\mathcal{A}$ if $\overline{\mathrm{I}_{\mathcal{A}}^{0}(E)}=\mathrm{I}_{\mathcal{A}}(E)$, and of local synthesis if $\overline{\mathrm{I}_{\mathcal{A}}^{0}(E)}=\overline{\mathrm{J}_{\mathcal{A}}^{0}(E)}$.

If $G$ is a compact group we let $\widehat{G}$ denote the dual object of $G$, a set of representatives, one from each unitary equivalence class, of irreducible continuous representations of $G$. If $\pi \in \widehat{G}$, we fix an orthonormal basis $\left\{\xi_{1}^{\pi}, \ldots, \xi_{d_{\pi}}^{\pi}\right\}$ for $\mathcal{H}_{\pi}$ and define

$$
\pi_{i j}: G \rightarrow \mathbb{C}, \quad \pi_{i j}(s)=\left\langle\pi(s) \xi_{j}^{\pi} \mid \xi_{i}^{\pi}\right\rangle,
$$

for $i, j=1, \ldots, d_{\pi}$. We recall the well-known fact that

$$
\mathcal{T}(G)=\operatorname{span}\left\{\pi_{i j}: \pi \in \widehat{G}, i, j=1, \ldots, d_{\pi}\right\}
$$

is uniformly dense in $\mathcal{C}(G)$. 
If $G$ is not compact, we let $\widehat{G}_{f}$ denote the finite-dimensional part of the dual object. We let $\mathcal{T}_{f}(G)$ be defined as the span of matrix coefficients of $\widehat{G}_{f}$, just as above. The almost periodic compactification is the compact group

$$
G^{\text {ap }}=\overline{\left\{(\pi(s))_{\pi \in \widehat{G}_{f}}: s \in G\right\}} \subset \prod_{\pi \in \widehat{G}_{f}} \overline{\pi(G)} .
$$

There is a canonical identification $\mathcal{T}\left(G^{\text {ap }}\right) \cong \mathcal{T}_{f}(G)$. We say $G$ is maximally almost periodic, or [MAP], if $\widehat{G}_{f}$, or equivalently $\mathcal{T}_{f}(G)$, separates the points of $G$. If $K$ is a compact subgroup of $G$, we say $G$ is $[\mathrm{MAP}]_{K}$ if the map $k \mapsto(\pi(k))_{\pi \in \widehat{G}_{f}}: K \rightarrow G^{\text {ap }}$ is injective. Clearly, a [MAP] group is [MAP] $K$ for any compact subgroup $K$.

The following is an adaptation of [31, Thm. 3.1]. A change in perspective allows us to gain not only more general, but finer results than in [31.

THEOREM 1.4. Let $G$ be a locally compact group, $K$ a compact subgroup so $G$ is $[\mathrm{MAP}]_{K}$, and $\mathcal{A}(G)$ be as in Section 1.1 and additionally satisfy $\mathcal{T}_{f}(G) \mathcal{A}(G) \subset \mathcal{A}(G)$. If $E$ is a closed subset of $G / K$ let $E^{*}=\{s \in G$ : $s K \in E\}$. Then

(i) $\Gamma \mathrm{I}_{\mathcal{A}(G)}\left(E^{*}\right)=\mathrm{I}_{\mathcal{A}(G / K)}(E)$,

(ii) $\mathrm{I}_{\mathcal{A}(G)}\left(E^{*}\right)$ is the closed ideal generated by $N \mathrm{I}_{\mathcal{A}(G / K)}(E)$.

Note that if $G$ is compact then in the case of Section 1.2 we have $E^{*}=$ $\left\{(s, t) \in G \times G: s t^{-1} \in E\right\}$ via the identification 1.1.

Proof. We will let $\mathrm{I}\left(E^{*}\right)=\mathrm{I}_{\mathcal{A}(G)}\left(E^{*}\right)$ and $\mathrm{I}(E)=\mathrm{I}_{\mathcal{A}(G / K)}(E)$ below.

(i) It is clear that

$$
\Gamma \mathrm{I}\left(E^{*}\right) \subset \mathrm{I}(E) \quad \text { and } \quad N \mathrm{I}(E) \subset \mathrm{I}\left(E^{*}\right) .
$$

Thus

$$
\mathrm{I}(E)=\Gamma \circ N \mathrm{I}(E) \subset \Gamma \mathrm{I}\left(E^{*}\right) \subset \mathrm{I}(E) .
$$

(ii) Let $w \in \mathrm{I}\left(E^{*}\right)$. For each $\pi$ in $\widehat{G}_{f}$ we define "matrix-valued" functions $w^{\pi}, \tilde{w}^{\pi}: G \rightarrow \mathcal{B}\left(\mathcal{H}_{\pi}\right)$ by

$$
w^{\pi}(s)=\int_{K} w(s k) \pi(k) d k, \quad \tilde{w}^{\pi}(s)=\pi(s) w^{\pi}(s) .
$$

Then for any $i, j=1, \ldots, d_{\pi}$ we let $w_{i j}^{\pi}=\left\langle w^{\pi}(\cdot) \xi_{j}^{\pi} \mid \xi_{i}^{\pi}\right\rangle$ and we notice that

$$
w_{i j}^{\pi}=\left.\pi_{i j}\right|_{K} \cdot w
$$

where $f \cdot w=\int_{G} f(k) k \cdot w d k$ for any $f$ in $\mathrm{L}^{1}(K)$. We note that since $w \in$ $\mathrm{I}\left(E^{*}\right)$, we have $k \cdot w \in \mathrm{I}\left(E^{*}\right)$ for any $k$ in $K$ and hence $f \cdot w \in \mathrm{I}\left(E^{*}\right)$ for any 
$f$ in $\mathrm{L}^{1}(K)$. Thus each $w_{i j}^{\pi}$ is in $\mathrm{I}\left(E^{*}\right)$. Now for any $s$ in $G$ and $i, j=1, \ldots, d_{\pi}$ we have

$$
\tilde{w}_{i j}^{\pi}(s)=\sum_{l=1}^{d_{\pi}} \pi_{i l}(s) w_{l j}^{\pi}(s), \quad \text { i.e. } \quad \tilde{w}_{i j}^{\pi}=\sum_{k=1}^{d_{\pi}} \pi_{i k} w_{l j}^{\pi} .
$$

Since $\mathcal{T}_{f}(G) \mathcal{A}(G) \subset \mathcal{A}(G)$ it follows that each $\tilde{w}_{i j}^{\pi}$ is in $\mathrm{I}\left(E^{*}\right)$. However, it is easily seen that $\tilde{w}^{\pi}(s k)=\tilde{w}^{\pi}(s)$ for any $s$ in $G$ and $k$ in $K$, so each $\tilde{w}_{i j}^{\pi}$ is also in $\mathcal{A}(G: K)$. Thus

$$
\tilde{w}_{i j}^{\pi} \in \mathrm{I}\left(E^{*}\right) \cap \mathcal{A}(G: K)=N \mathrm{I}(E) .
$$

Now we use the relation $w^{\pi}(s)=\pi\left(s^{-1}\right) \tilde{w}^{\pi}(s)$, for $s$ in $G$, to see that for each $i, j$ we have

$$
w_{i j}^{\pi}=\sum_{l=1}^{d_{\pi}} \check{\pi}_{i l} \tilde{w}_{l j}^{\pi}=\sum_{l=1}^{d_{\pi}} \bar{\pi}_{l i} \tilde{w}_{l j}^{\pi}
$$

so $w_{i j}^{\pi}=\left.\pi_{i j}\right|_{K} \cdot w$ lies in the ideal generated by $N \mathrm{I}(E)$.

Since $G$ is $[\mathrm{MAP}]_{K}$, we may regard $K$ as a closed subgroup of $G^{\text {ap }}$. We see that $\left.\mathcal{T}_{f}(G)\right|_{K}=\left.\mathcal{T}\left(G^{\text {ap }}\right)\right|_{K}$ is a conjugation-closed point-separating subalgebra of $\mathcal{C}(K)$, thus is uniformly dense in $\mathcal{C}(K)$, and hence norm dense in $\mathrm{L}^{1}(K)$. Thus there is a bounded approximate identity $\left(f_{\beta}\right)$ for $\mathrm{L}^{1}(K)$ with each $f_{\beta}$ in $\left.\mathcal{T}_{f}(G)\right|_{K}$. Then for each $\beta$ we have

$$
f_{\beta} \cdot w \in \operatorname{span}\left\{\left.\pi_{i j}\right|_{K} \cdot w: \pi \in \widehat{G} \text { and } i, j=1, \ldots, d_{\pi}\right\}
$$

and is thus in the ideal generated by $N \mathrm{I}(E)$. Hence, since $\mathcal{A}(G)$ is an essential $\mathrm{L}^{1}(K)$-module, we find that $w=\lim _{\beta} f_{\beta} \cdot w$ and thus $w$ is in the closed ideal generated by $N \mathrm{I}(E)$.

For any subalgebra $\mathcal{B}$ of a commutative normed algebra $\mathcal{A}$, we let

$\langle\mathcal{B}\rangle=\overline{\operatorname{span}}\left\{a b_{1}+b_{2}: a \in \mathcal{A}, b_{1}, b_{2} \in \mathcal{B}\right\}$ and $\mathcal{B}^{2}=\operatorname{span}\{a b: a, b \in \mathcal{B}\}$.

Corollary 1.5. With $G, K, \mathcal{A}(G), E \subset G / K$ and $E^{*} \subset G$ as in Theorem 1.4, we have:

(i) $\mathrm{I}_{\mathcal{A}(G)}\left(E^{*}\right)$ has a bounded approximate identity (b.a.i.) if and only if $\mathrm{I}_{\mathcal{A}(G / K)}(E)$ has a b.a.i.;

(ii) $\overline{\mathrm{I}_{\mathcal{A}(G)}^{0}\left(E^{*}\right)}=\mathrm{I}_{\mathcal{A}(G)}\left(E^{*}\right)$ if and only if $\overline{\mathrm{I}_{\mathcal{A}(G / K)}^{0}(E)}=\mathrm{I}_{\mathcal{A}(G / K)}(E)$;

(ii') $\overline{\mathrm{I}_{\mathcal{A}(G)}^{0}\left(E^{*}\right)}=\overline{\mathrm{J}_{\mathcal{A}(G)}^{0}\left(E^{*}\right)}$ if and only if $\overline{\mathrm{I}_{\mathcal{A}(G / K)}^{0}(E)}=\overline{\mathrm{J}_{\mathcal{A}(G / K)}^{0}(E)}$;

(iii) $\overline{\mathrm{I}_{\mathcal{A}(G)}\left(E^{*}\right)^{2}}=\mathrm{I}_{\mathcal{A}(G)}\left(E^{*}\right)$ if and only if $\overline{\mathrm{I}_{\mathcal{A}(G / K)}(E)^{2}}=\mathrm{I}_{\mathcal{A}(G / K)}(E)$.

Proof. We let $\mathrm{I}\left(E^{*}\right)=\mathrm{I}_{\mathcal{A}(G)}\left(E^{*}\right), \mathrm{I}(E)=\mathrm{I}_{\mathcal{A}(G / K)}(E)$, etc.

(i) If $\left(f_{\alpha}\right)$ is a b.a.i. for $\mathrm{I}(E)$, then $\left(N f_{\alpha}\right)$ is a b.a.i. for the subalgebra $N \mathrm{I}(E)$. It is readily checked that $\left(N f_{\alpha}\right)$ is a b.a.i. for $\langle N \mathrm{I}(E)\rangle$. 
If $\left(w_{\alpha}\right)$ is a b.a.i. for $\mathrm{I}\left(E^{*}\right)$, then $\left(\Gamma w_{\alpha}\right)$ is a b.a.i. for $\mathrm{I}(E)$. Indeed, $\Gamma \circ N=$ id and, since $P$ is an idempotent, we have the following "expectation property":

$$
\Gamma(w N f)=\Gamma(w) f
$$

for $w \in \mathcal{A}(G \times G)$ and $f \in \mathcal{A}(G / K)$. Thus if $f$ in $\mathrm{I}(E)$ then

$$
\Gamma\left(w_{\alpha}\right) f-f=\Gamma\left(w_{\alpha} N f-N f\right) \rightarrow 0 .
$$

(ii) \& (ii') It is clear that

$$
f \in \mathrm{I}^{0}(E) \Leftrightarrow N f \in \mathrm{I}^{0}\left(E^{*}\right) \text { and } f \in \mathrm{J}^{0}(E) \Leftrightarrow N f \in \mathrm{J}^{0}\left(E^{*}\right) .
$$

It can then be proved exactly as in Theorem 1.4 that

$$
\left\langle N \mathrm{I}^{0}(E)\right\rangle=\overline{\mathrm{I}^{0}\left(E^{*}\right)}, \quad\left\langle N \mathrm{~J}^{0}(E)\right\rangle=\overline{\mathrm{J}^{0}\left(E^{*}\right)} .
$$

Indeed, it is sufficient to note that if $\operatorname{supp}(f)$ is compact then so too is $\operatorname{supp}\left(\left(\left.\pi_{i j}\right|_{K} \cdot f\right)\right)$ for each $\pi \in \widehat{G}_{f}$ and $i, j=1, \ldots, d_{\pi}$.

(iii) If $\overline{\mathrm{I}(E)^{2}}=\mathrm{I}(E)$, then $N\left(\mathrm{I}(E)^{2}\right)=(N \mathrm{I}(E))^{2}$ is dense in $N \mathrm{I}(E)$. Hence we have

$$
\mathrm{I}\left(E^{*}\right)=\langle N \mathrm{I}(E)\rangle=\left\langle(N \mathrm{I}(E))^{2}\right\rangle=\overline{\langle N \mathrm{I}(E)\rangle^{2}}=\overline{\mathrm{I}\left(E^{*}\right)^{2}} .
$$

Conversely, if $\overline{\mathrm{I}\left(E^{*}\right)^{2}}=\mathrm{I}\left(E^{*}\right)$ then from the theorem above we have

$$
\mathrm{I}\left(E^{*}\right)=\overline{\mathrm{I}\left(E^{*}\right)^{2}}=\overline{\mathrm{I}\left(E^{*}\right) N \mathrm{I}(E)} \text {. }
$$

But it follows from 1.5 that

$$
\mathrm{I}(E)=\Gamma \mathrm{I}\left(E^{*}\right)=\overline{\Gamma\left(\mathrm{I}\left(E^{*}\right) N \mathrm{I}(E)\right)}=\overline{\Gamma \mathrm{I}\left(E^{*}\right) \mathrm{I}(E)}=\overline{\mathrm{I}(E)^{2}} \subset \mathrm{I}(E),
$$

whence $\mathrm{I}(E)=\overline{\mathrm{I}(E)^{2}}$.

\section{Some subalgebras of Fourier algebras}

2.1. The algebra $\mathrm{A}_{\Delta}(G)$. In this section, we will always let $G$ denote a compact group. We have the following characterisation of the Fourier algebra in [14, (34.4)]: for $f \in \mathcal{C}(G)$,

$$
f \in \mathrm{A}(G) \Leftrightarrow\|f\|_{\mathrm{A}}=\sum_{\pi \in \widehat{G}} d_{\pi}\|\hat{f}(\pi)\|_{1}<\infty
$$

where $\hat{f}(\pi)=\int_{G} f(s) \bar{\pi}(s) d s$ and $\|\cdot\|_{1}$ denotes the trace class norm. We also recall the following orthogonality relations [14, (27.19)]: if $\pi, \sigma \in \widehat{G}$ then in the notation of $(1.3)$ we have

$$
\int_{G} \pi_{i j}(s) \overline{\sigma_{k l}(s)} d s=\frac{1}{d_{\pi}} \delta_{\pi \sigma} \delta_{j l} \delta_{i k}
$$

where $i, j=1, \ldots, d_{\pi}, k, l=1, \ldots, d_{\sigma}$ and each $\delta_{\alpha \beta}$ is the Kronecker $\delta$ symbol. 
We first wish to characterise $\mathrm{A}_{\Delta}(G)$, as defined in the previous section. We will make use of the following lemma. For $\pi, \sigma \in \widehat{G}$ we let $\pi \times \sigma \in \widehat{G \times G}$ be the Kronecker product representation. Also, we let $N: \mathcal{C}(G) \rightarrow \mathcal{C}(G \times G)$ be given by $N f(s, t)=f\left(s t^{-1}\right)$ for $(s, t)$ in $G \times G$, as suggested by 1.2 .

Lemma 2.1. For any $f \in \mathcal{C}(G)$ and $\pi \in \widehat{G}$, we have

$$
\|\widehat{N f}(\bar{\pi} \times \pi)\|_{p}=\frac{1}{\sqrt{d_{\pi}}}\|\hat{f}(\pi)\|_{2}
$$

where $\|\cdot\|_{p}$ is the Schatten $p$-norm for $1 \leq p \leq \infty$.

Proof. We first note that

$$
\begin{aligned}
\widehat{N f}(\bar{\pi} \times \pi) & =\int_{G} \int_{G} f\left(s t^{-1}\right) \pi(s) \otimes \bar{\pi}(t) d s d t \\
& =\int_{G} \int_{G} f(s) \pi(s t) \otimes \bar{\pi}(t) d s d t \\
& =\left[\int_{G} f(s) \pi(s) \otimes \bar{\pi}(e) d s\right] \circ\left[\int_{G} \pi(t) \otimes \bar{\pi}(t) d t\right] \\
& =\left[\hat{f}(\bar{\pi}) \otimes I_{\mathcal{H}_{\bar{\pi}}}\right] \circ P_{1}
\end{aligned}
$$

where $P_{1}$ is a rank 1 projection, as we shall see below. Indeed, the Schur orthogonality relations [14, (27.30)] tell us that $\pi \otimes \bar{\pi}$ contains the trivial representation 1 with multiplicity 1 . Thus when we decompose into irreducibles $\pi \otimes \bar{\pi} \cong \bigoplus_{\sigma \in \widehat{G}} m_{\sigma} \cdot \sigma$, we obtain

$$
\int_{G} \pi(t) \otimes \bar{\pi}(t) d t \cong \bigoplus_{\sigma \in \widehat{G}} m_{\sigma} \cdot \int_{G} \sigma(t) d t
$$

where $\int_{G} \sigma(t) d t=0$ unless $\sigma=1$, and hence the formula above reduces to a rank 1 projection, $P_{1}$. Let $\left\{\xi_{1}, \ldots, \xi_{d_{\pi}}\right\}$ be an orthonormal basis for $\mathcal{H}_{\pi}$; we claim that

$$
P_{1}=\langle\cdot \mid \eta\rangle \eta \quad \text { where } \quad \eta=\frac{1}{\sqrt{d_{\pi}}} \sum_{k=1}^{d_{\pi}} \xi_{k} \otimes \bar{\xi}_{k} .
$$

Indeed, for any $d_{\pi} \times d_{\pi}$ unitary matrix $U$ we have $\sum_{k=1}^{d_{\pi}} U \xi_{k} \otimes \overline{U \xi}_{k}=$ $\sum_{k=1}^{d_{\pi}} \xi_{k} \otimes \bar{\xi}_{k}$. Hence $\eta$ is a norm 1 vector, invariant for $\pi \otimes \bar{\pi}$, and the formula for $P_{1}$ follows.

Thus

$$
\widehat{N f}(\bar{\pi} \otimes \pi)=\left[\hat{f}(\bar{\pi}) \otimes I_{\mathcal{H}_{\bar{\pi}}}\right] \circ P_{1}=\langle\cdot \mid \eta\rangle\left[\hat{f}(\bar{\pi}) \otimes I_{\mathcal{H}_{\bar{\pi}}}\right] \eta
$$

and, using the standard formula for rank 1 operators, $\|\langle\cdot \mid \zeta\rangle \vartheta\|_{p}=\|\zeta\|\|\vartheta\|$, we obtain

$$
\|\widehat{N f}(\bar{\pi} \otimes \pi)\|_{p}=\left\|\left[\hat{f}(\bar{\pi}) \otimes I_{\mathcal{H}_{\bar{\pi}}}\right] \eta\right\|_{\mathcal{H}_{\pi} \otimes^{2} \mathcal{H}_{\bar{\pi}}} .
$$


Letting $\hat{f}(\bar{\pi})_{k l}=\left\langle\hat{f}(\bar{\pi}) \xi_{l} \mid \xi_{k}\right\rangle$ we have

$$
\left[\hat{f}(\bar{\pi}) \otimes I_{\mathcal{H}_{\bar{\pi}}}\right] \eta=\frac{1}{\sqrt{d_{\pi}}} \sum_{k=1}^{d_{\pi}}\left[\hat{f}(\bar{\pi}) \xi_{k}\right] \otimes \bar{\xi}_{k}=\frac{1}{\sqrt{d_{\pi}}} \sum_{k=1}^{d_{\pi}} \sum_{l=1}^{d_{\pi}} \hat{f}(\bar{\pi})_{k l} \xi_{k} \otimes \bar{\xi}_{l} .
$$

Since $\left\{\xi_{l} \otimes \bar{\xi}_{k}: k, l=1, \ldots, d_{\pi}\right\}$ is an orthonormal basis for $\mathcal{H}_{\pi} \otimes^{2} \mathcal{H}_{\bar{\pi}}$ we obtain

$$
\left\|\left[\hat{f}(\bar{\pi}) \otimes I_{\mathcal{H}_{\bar{\pi}}}\right] \eta\right\|_{\mathcal{H}_{\pi} \otimes^{2} \mathcal{H}_{\bar{\pi}}}^{2}=\frac{1}{d_{\pi}} \sum_{k=1}^{d_{\pi}} \sum_{l=1}^{d_{\pi}}\left|\hat{f}(\bar{\pi})_{k l}\right|^{2}=\frac{1}{d_{\pi}}\|\hat{f}(\bar{\pi})\|_{2}^{2} .
$$

The result is obtained by combining (2.4) with (2.5) and the fact that $\|\hat{f}(\bar{\pi})\|_{2}=\|\hat{f}(\pi)\|_{2}$.

We now obtain a characterisation of $\mathrm{A}_{\Delta}(G)$ in the spirit of (2.1).

Theorem 2.2. If $f \in \mathcal{C}(G)$, then

$$
f \in \mathrm{A}_{\Delta}(G) \Leftrightarrow \sum_{\pi \in \widehat{G}} d_{\pi}^{3 / 2}\|\hat{f}(\pi)\|_{2}<\infty .
$$

Moreover, the latter quantity is

$$
\|f\|_{\mathrm{A}_{\Delta}}=\inf \left\{\|w\|_{\mathrm{A}}: w \in \mathrm{A}(G \times G) \text { with } \Gamma w=f\right\} .
$$

Proof. Since $N: \mathrm{A}_{\Delta}(G) \rightarrow \mathrm{A}(G \times G: \Delta) \subset \mathrm{A}(G \times G)$ is an isometry we have

$$
f \in \mathrm{A}_{\Delta}(G) \Leftrightarrow N f \in \mathrm{A}(G \times G),
$$

in which case $\|f\|_{\mathrm{A}_{\Delta}}=\|N f\|_{\mathrm{A}}$. Recall that $\widehat{G \times G}=\{\pi \times \sigma: \pi, \sigma \in \widehat{G}\}$ (see [14, (27.43)], for example). Note that analogous computations to (2.3), combined with 2.2 , show that

$$
\widehat{N f}(\pi \times \sigma)=0 \quad \text { if } \sigma \neq \bar{\pi} .
$$

We see that $N f \in \mathrm{A}(G \times G)$ exactly when

$$
\begin{aligned}
\|N f\|_{\mathrm{A}} & =\sum_{(\sigma, \pi) \in \widehat{G} \times \widehat{G}} d_{\sigma} d_{\pi}\|\widehat{N f}(\sigma \times \pi)\|_{1} & & \text { by 2.1. } \\
& =\sum_{\pi \in \widehat{G}} d_{\pi}^{2}\|\widehat{N f}(\bar{\pi} \times \pi)\|_{1} & & \text { by 2.6. } \\
& =\sum_{\pi \in \widehat{G}} d_{\pi}^{3 / 2}\|\hat{f}(\pi)\|_{2} & & \text { by Lemma 2.1. }
\end{aligned}
$$

and the latter quantity is finite.

We prove some consequences of the result above. Let us note for any $d \times d$ matrix the well-known inequalities

$$
\frac{1}{\sqrt{d}}\|A\|_{1} \leq\|A\|_{2} \leq\|A\|_{1} .
$$


These inequalities are sharp with scalar matrices $\alpha I$ serving as the extreme case for the left inequality, and rank 1 matrices serving for the extreme case on the right.

We let $\mathrm{ZL}^{1}(G)$ be the centre of the convolution algebra $\mathrm{L}^{1}(G)$.

Corollary 2.3. We have $\mathrm{A}(G) \cap \mathrm{ZL}^{1}(G)=\mathrm{A}_{\Delta}(G) \cap \mathrm{ZL}^{1}(G)$ with $\|f\|_{\mathrm{A}}=\|f\|_{\mathrm{A}_{\Delta}}$ for $f$ in this space.

Proof. It is well-known that $f \in \mathrm{ZL}^{1}(G)$ only if $\hat{f}(\pi)=\alpha_{f, \pi} I_{\mathcal{H}_{\pi}}$ for some scalar $\alpha_{f, \pi}$, for each $\pi$ in $\widehat{G}$. Thus by the left extreme case of 2.7 we have

$$
\sum_{\pi \in \widehat{G}} d_{\pi}^{3 / 2}\|\hat{f}(\pi)\|_{2}=\sum_{\pi \in \widehat{G}} d_{\pi}\|\hat{f}(\pi)\|_{1} .
$$

Hence it follows that $f \in \mathrm{A}(G) \cap \mathrm{ZL}^{1}(G)$ if and only if $f \in \mathrm{A}_{\Delta}(G) \cap \mathrm{ZL}^{1}(G)$, with $\|f\|_{\mathrm{A}}=\|f\|_{\mathrm{A}_{\Delta}}$.

Corollary 2.4. We have $\mathrm{A}_{\Delta}(G)=\mathrm{A}(G)$ if and only if $G$ admits an abelian subgroup of finite index.

Proof. We invoke the well-known result of [21] that

$G$ admits an abelian subgroup of finite index $\Leftrightarrow d_{G}=\sup _{\pi \in \widehat{G}} d_{\pi}<\infty$.

Now if $d_{G}<\infty$, then for any $u$ in $\mathrm{A}(G)$, by (2.7),

$$
\sum_{\pi \in \widehat{G}} d_{\pi}^{3 / 2}\|\hat{u}(\pi)\|_{2} \leq d_{G}^{1 / 2} \sum_{\pi \in \widehat{G}} d_{\pi}\|\hat{u}(\pi)\|_{1}<\infty
$$

so $u \in \mathrm{A}_{\Delta}(G)$. Conversely, if $\mathrm{A}(G)=\mathrm{A}_{\Delta}(G)$, then since $\|\cdot\|_{\mathrm{A}} \leq\|\cdot\|_{\mathrm{A}_{\Delta}}$, the open mapping theorem provides us with a constant $K$ such that $\|\cdot\|_{\mathrm{A}_{\Delta}} \leq$ $K\|\cdot\|_{\mathrm{A}}$. For any $\pi$ in $\widehat{G}$ we let $\pi_{11}$ be as in 1.3 . We have

$$
\hat{\pi}_{11}(\sigma)=\int_{G}\left\langle\pi(s) \xi_{1}^{\pi} \mid \xi_{1}^{\pi}\right\rangle \bar{\sigma}(s) d s= \begin{cases}\left(1 / d_{\pi}\right)\left\langle\cdot \mid \bar{\xi}_{1}^{\pi}\right\rangle \bar{\xi}_{1}^{\pi} & \text { if } \sigma=\pi, \\ 0 & \text { otherwise. }\end{cases}
$$

Thus by the rank 1 case of 2.7 we have

$$
d_{\pi}^{3 / 2}\left\|\hat{\pi}_{11}(\pi)\right\|_{1}=d_{\pi}^{3 / 2}\left\|\hat{\pi}_{11}(\pi)\right\|_{2}=\left\|\hat{\pi}_{11}\right\|_{\mathrm{A}_{\Delta}} \leq K\left\|\hat{\pi}_{11}\right\|_{\mathrm{A}}=K d_{\pi}\left\|\hat{\pi}_{11}(\pi)\right\|_{1},
$$

so $d_{\pi} \leq K^{2}$. Thus $d_{G} \leq K^{2}<\infty$.

We remark that despite the identification $(G \times G) / \Delta \cong G$, the result above tells us that the Fourier algebra over this coset space, $\mathrm{A}(G \times G / \Delta)$ $\cong \mathrm{A}_{\Delta}(G)$, is not naturally isomorphic to $\mathrm{A}(G)$. We will see in Section 3 that $\mathrm{A}_{\Delta}(G)$ can fail to be operator weakly amenable, while $\mathrm{A}(G)$ is always operator weakly amenable. 
2.2. Some subalgebras of $\mathrm{A}_{\Delta}(G)$. Let us begin with a variant of Proposition 1.2 .

Proposition 2.5.

(i) There is a completely isometric identification

$$
\mathrm{A}_{\Delta}(G) \widehat{\otimes} \mathrm{A}_{\Delta}(H) \cong \mathrm{A}_{\Delta}(G \times H)
$$

given on elementary tensors by $f \otimes g \mapsto f \times g$.

(ii) If $K$ is a closed subgroup of $G$, and $L$ is a closed subgroup of $H$, then we obtain a completely isometric identification

$$
\mathrm{A}_{\Delta}(G / K) \widehat{\otimes} \mathrm{A}_{\Delta}(H / L) \cong \mathrm{A}_{\Delta}(G \times H / K \times L) .
$$

Proof. (i) Using Proposition 1.2 , we have completely isometric identifications

$$
\begin{aligned}
\mathrm{A}_{\Delta}(G) \widehat{\otimes} \mathrm{A}_{\Delta}(H) & \cong \mathrm{A}\left(G \times G / \Delta_{G}\right) \widehat{\otimes} \mathrm{A}\left(H \times H / \Delta_{H}\right) \\
& \cong \mathrm{A}\left(G \times G \times H \times H / \Delta_{G} \times \Delta_{H}\right) .
\end{aligned}
$$

Moreover, we have a completely isometric identification

$$
\mathrm{A}_{\Delta}(G \times H) \cong \mathrm{A}\left(G \times H \times G \times H / \Delta_{G \times H}\right) .
$$

Thus we must show that

$$
\mathrm{A}\left(G \times G \times H \times H / \Delta_{G} \times \Delta_{H}\right) \cong \mathrm{A}\left(G \times H \times G \times H / \Delta_{G \times H}\right) .
$$

Let $\varsigma$ be the topological group isomorphism $\left(s_{1}, t_{1}, s_{2}, t_{2}\right) \mapsto\left(s_{1}, s_{2}, t_{1}, t_{2}\right)$ : $G \times H \times G \times H \rightarrow G \times G \times H \times H$. This map induces a completely isometric isomorphism $u \mapsto u \circ \varsigma$ from $\mathrm{A}(G \times G \times H \times H)$ to $\mathrm{A}(G \times H \times G \times H)$. Moreover, the following diagram commutes:

$$
\begin{gathered}
\mathrm{A}(G \times G \times H \times H) \underset{u \mapsto u \circ \varsigma^{-1}}{P_{\Delta_{G} \times \Delta_{H}} \mid} \mathrm{A}(G \times H \times G \times H) \\
\mathrm{A}\left(G \times G \times H \times H: \Delta_{G} \times \Delta_{H}\right) \underset{u \mapsto u \circ \varsigma}{\stackrel{u \mapsto u \circ \varsigma^{-1}}{\underset{u}{u}}} \mathrm{~A}\left(G \times H \times G \times H: \Delta_{G \times H}\right)
\end{gathered}
$$

Since both top row maps are complete isometries, and both $P_{\Delta_{G} \times \Delta_{H}}$ and $P_{\Delta_{G \times H}}$ are complete quotient maps, we deduce that the bottom row maps must each be complete quotient maps which are mutual inverses, hence complete isometries. By standard identifications, 2.8 follows.

(ii) This can be proved exactly as Proposition 1.2 using (i) above, in place of the identification $\mathrm{A}(G) \widehat{\otimes} \mathrm{A}(H) \cong \mathrm{A}(G \times H)$.

We now define a sequence of subalgebras of $\mathrm{A}(G)$.

Theorem 2.6. Let $\mathrm{A}_{\Delta^{1}}(G)=\mathrm{A}_{\Delta}(G)$ and for each $n \geq 1$ let

$$
\mathrm{A}_{\Delta^{n+1}}(G)=\Gamma\left(\mathrm{A}_{\Delta^{n}}(G \times G)\right) .
$$


(i) Each $\mathrm{A}_{\Delta^{n}}(G)$ is a subalgebra of $\mathrm{A}(G)$, which is closed under both left and right translations and for which all translations are complete isometries. Also, for each $n$, the map $\Gamma: \mathrm{A}_{\Delta^{n}}(G \times G) \rightarrow \mathrm{A}_{\Delta^{n+1}}(G)$ is a complete quotient map.

(ii) If $f \in \mathcal{C}(G)$, then

$$
f \in \mathrm{A}_{\Delta^{n}}(G) \Leftrightarrow \sum_{\pi \in \widehat{G}} d_{\pi}^{\left(2^{n}+1\right) / 2}\|\hat{f}(\pi)\|_{2}<\infty .
$$

Moreover, the latter quantity is the norm, $\|f\|_{\mathrm{A}_{\Delta^{n}}}$.

Proof. (i) We use induction. If the ascribed properties hold for $\mathrm{A}_{\Delta^{n}}(G)$, and we have

$$
\mathrm{A}_{\Delta^{n}}(G) \widehat{\otimes} \mathrm{A}_{\Delta^{n}}(G) \cong \mathrm{A}_{\Delta^{n}}(G \times G),
$$

then it follows from Proposition 1.3 (i) that $\mathrm{A}_{\Delta^{n+1}}(G)$ is a subalgebra of $\mathrm{A}(G)$, which is completely isometrically isomorphic to a completely contractively complemented subspace of $\mathrm{A}_{\Delta^{n}}(G \times G)$. It follows from Proposition 1.3(ii) that $\mathrm{A}_{\Delta^{n+1}}(G)$ is closed under left and right translations. The formula 2.9 holds for $n=1$ by Proposition 2.5(i). Moreover, if 2.9) holds for $n$, then we can use the proof of Proposition 2.5(ii), then (i), to see that (2.9) holds for $n+1$.

(ii) This follows exactly as the proof of Theorem 2.2, using Lemma 2.1 in the case $p=2$, and induction.

We note that a simple modification of Corollary 2.4 shows that if $G$ admits no abelian subgroup of finite index then $\left\{\mathrm{A}_{\Delta^{n}}(G)\right\}_{n=1}^{\infty}$ is a properly nested sequence of subalgebras of $\mathrm{A}(G)$. We also note that it follows from Proposition 1.1(i) that $\Sigma_{\mathrm{A}_{\Delta}(G)} \cong G$. We suspect the same holds for each $\mathrm{A}_{\Delta^{n}}(G)(n \geq 2)$, but have not been able to prove it. We cannot copy the method of proof of [6. Thm. 4.1] which we cited in Proposition 1.1(i) to obtain $\Sigma_{\mathrm{A}_{\Delta^{2}}(G)} \cong G$. Indeed, $\Delta$ is not a set of spectral synthesis for $\mathrm{A}_{\Delta}(G \times G)$ when $G$ is a nonabelian connected Lie group, by (3.1) below.

\section{Amenability properties}

3.1. Failure of operator weak amenability of $\mathrm{A}_{\Delta}(G)$. We adapt arguments from [17, Thm. 7.2 \& Cor. 7.3]. We also use the Fourier series of any $f$ in $\mathcal{T}(G)$ (with $\mathcal{T}(G)$ defined in 1.4 ):

$$
f(s)=\sum_{\pi \in \widehat{G}} d_{\pi} \sum_{i, j=1}^{d_{\pi}} \hat{f}(\pi)_{i j} \pi_{i j}(s)=\sum_{\pi \in \widehat{G}} d_{\pi} \operatorname{trace}\left[\hat{f}(\pi) \pi(s)^{\mathrm{t}}\right]
$$

where $\hat{f}(\pi)_{i j}=\left\langle\hat{f}(\pi) \xi_{j}^{\pi} \mid \xi_{i}^{\pi}\right\rangle$ and $A^{\mathrm{t}}$ is the transpose of a matrix $A$. This is a variant of the formula given in [14, (34.1)]. 
THEOREM 3.1. If $G$ is a nonabelian connected compact Lie group, then $\mathrm{A}_{\Delta}(G) \cong \mathrm{A}(G \times G / \Delta)$ is not operator weakly amenable.

Proof. We will establish that $\mathrm{A}_{\Delta^{2}}(G)$ admits a point derivation at $e$. This implies that $\overline{\mathrm{I}_{\mathrm{A}_{\Delta^{2}}(G)}(\{e\})^{2}} \neq \mathrm{I}_{\mathrm{A}_{\Delta^{2}}(G)}(\{e\})$. Indeed, the point derivation will vanish on $\mathrm{I}_{\mathrm{A}_{\Delta^{2}}(G)}(\{e\})^{2}$, but not on $\mathrm{I}_{\mathrm{A}_{\Delta^{2}}(G)}(\{e\})$. Hence it follows from the construction of $\mathrm{A}_{\Delta^{2}}(G)$ (Theorem 2.6), and Corollary 1.5(iii), that

$$
\overline{\mathrm{I}_{\mathrm{A}_{\Delta}(G \times G)}(\Delta)^{2}} \neq \mathrm{I}_{\mathrm{A}_{\Delta}(G \times G)}(\Delta) .
$$

This condition implies that $\mathrm{A}_{\Delta}(G)$ is not operator weakly amenable by [12, Thm. 3.2], which was shown to be a valid characterisation of operator weak amenability in [29].

It has been shown in 24] that under the assumptions given, there is a subgroup $T \cong \mathbb{T}$ in $G$ such that for any $\pi$ in $\widehat{G}$,

$$
\left.\pi\right|_{T} \cong \bigoplus_{k=1}^{d_{\pi}} \chi_{n_{k}} \quad \text { with each }\left|n_{k}\right|<d_{\pi}
$$

where $\chi_{n}(z)=z^{n}$ for $n$ in $\mathbb{Z} \cong \widehat{\mathbb{T}}$. We let $\theta \mapsto t_{\theta}:(-\pi, \pi] \rightarrow T$ be the parameterisation of $T$ which corresponds to $\theta \mapsto e^{i \theta}:(-\pi, \pi] \rightarrow \mathbb{T}$. For each $\pi$ in $\widehat{G}$ we can choose an orthonormal basis for $\mathcal{H}_{\pi}$ with respect to which $\pi\left(t_{\theta}\right)=\operatorname{diag}\left(e^{i n_{1} \theta}, \ldots, e^{i n_{d_{\pi}} \theta}\right)$; and it follows by elementary estimates that

$$
\frac{\left\|\pi\left(t_{\theta}\right)^{\mathrm{t}}-I\right\|}{\theta}=\max _{k=1, \ldots, d_{\pi}} \frac{\left|e^{i n_{k} \theta}-1\right|}{|\theta|}<d_{\pi}
$$

for $\theta$ in a neighbourhood of 0 .

For $f$ in $\mathcal{T}(G)$ we have

$$
\frac{f\left(t_{\theta}\right)-f(e)}{\theta}=\sum_{\pi \in \widehat{G}} d_{\pi} \operatorname{trace}\left[\hat{f}(\pi) \frac{\pi\left(t_{\theta}\right)^{\mathrm{t}}-I}{\theta}\right] .
$$

We note that for small $\theta$ we have, using (2.7)

$$
\left|\frac{f\left(t_{\theta}\right)-f(e)}{\theta}\right| \leq \sum_{\pi \in \widehat{G}} d_{\pi}\|\hat{f}(\pi)\|_{1} \frac{\left\|\pi\left(t_{\theta}\right)^{\mathrm{t}}-I\right\|}{|\theta|} \leq \sum_{\pi \in \widehat{G}} d_{\pi}^{5 / 2}\|\hat{f}(\pi)\|_{2}
$$

where the last quantity is $\|f\|_{\mathrm{A}_{\Delta^{2}}}$, by Theorem 2.6(ii). Hence, since each $\lim _{n \rightarrow \infty}\left(\pi\left(t_{\theta}\right)-I\right) / \theta$ exists, it follows that

$$
d(f)=\left.\frac{d}{d \theta}\right|_{\theta=0} f\left(t_{\theta}\right)=\lim _{\theta \rightarrow 0} \frac{f\left(t_{\theta}\right)-f(e)}{\theta}
$$

exists, and $|d(f)| \leq\|f\|_{\mathrm{A}_{\Delta^{2}}}$ for $f$ in $\mathcal{T}(G)$. Hence $d$ extends to a contractive point derivation on $\mathrm{A}_{\Delta^{2}}(G)$ at $e$.

We remark that Johnson [17, Cor. 7.3] showed that the point derivation $d: \mathcal{T}(G) \rightarrow \mathbb{C}$ extends to a bounded map on $\mathrm{A}_{\gamma}(G)$ (see Section 4 below), 
and hence established that $\mathrm{A}(G)$ is not weakly amenable. Since $\Delta$ is a set of spectral synthesis for $\mathrm{A}(G \times G)$, we can proceed as in the first paragraph of the proof above to see that $d$ cannot be extended to $\mathrm{A}_{\Delta}(G)$.

As an application of the above result, we obtain a new set of nonsynthesis.

Corollary 3.2. Let $G$ be a compact connected nonabelian Lie group. Then $\left(\Delta_{G} \times \Delta_{G}\right) \Delta_{G \times G}$ fails spectral synthesis for $\mathrm{A}(G \times G \times G \times G)$.

Proof. It follows from (3.1) that $\Delta_{G}$ is not a set of spectral synthesis for $\mathrm{A}_{\Delta}(G \times G)$. We then appeal to Proposition 2.5(ii) to see that

$$
\mathrm{A}_{\Delta}(G) \hat{\otimes} \mathrm{A}_{\Delta}(G) \cong \mathrm{A}_{\Delta}(G \times G) \cong \mathrm{A}\left(G \times G \times G \times G / \Delta_{G \times G}\right) .
$$

In the identification (1.1) we have $\Delta_{G} \cong\left\{(s, s, e, e) \Delta_{G \times G}: s \in G\right\}$. It then follows from Corollary 1.5 (iii) that

$$
\Delta_{G}^{*} \cong\left(\Delta_{G} \times\{1\}\right) \Delta_{G \times G}=\left(\Delta_{G} \times \Delta_{G}\right) \Delta_{G \times G}
$$

is not spectral for $\mathrm{A}(G \times G \times G \times G)$.

3.2. Failure of operator weak amenability of $\mathrm{A}(G / K)$. Let us now turn our attention to general locally compact groups. We will let $G$ denote a locally compact group. Let us collect some useful facts.

LEMma 3.3. Let $H$ be a closed subgroup of $G$ and $K$ be a compact subgroup of $H$. Then the restriction map $\left.u \mapsto u\right|_{H}$ maps $\mathrm{A}(G: K)$ onto $\mathrm{A}(H: K)$.

Proof. This is [11, Lemma 3.6(ii)].

LEMma 3.4. Let $N$ be a compact normal subgroup of $G$, and $q: G \rightarrow$ $G / N$ be the canonical quotient map. If $\tilde{K}$ is a compact subgroup of $G / N$ and $K=q^{-1}(\tilde{K})$, then the algebras $\mathrm{A}(G: K)$ and $\mathrm{A}(G / N: \tilde{K})$ are completely isometrically isomorphic.

Proof. The map $u \mapsto u \circ q: \mathrm{A}(G / N) \rightarrow \mathrm{A}(G)$ is a complete isometry with range $\mathrm{A}(G: N)$. If $u \in \mathrm{A}(G / N: \tilde{K})$, then for $s$ in $G$ and $k$ in $K$ we have

$$
u \circ q(s k)=u(q(s) q(k))=u(q(s))=u \circ q(s)
$$

so $u \circ q \in \mathrm{A}(G: K)$. Conversely, let $v \in \mathrm{A}(G: K) \subset \mathrm{A}(G: N)$. Let $u$ in $\mathrm{A}(G / N)$ be such that $v=u \circ q$. For any $\tilde{s}$ in $G / N$ and $\tilde{k} \in \tilde{K}$, find $s \in G$ and $k \in K$ so $q(s)=\tilde{s}$ and $q(k)=\tilde{k}$. We have

$$
u(\tilde{s} \tilde{k})=u \circ q(s k)=v(s k)=v(s)=u(\tilde{s})
$$

so $u \in \mathrm{A}(G / N: \tilde{K})$. 
We now obtain a generalisation of Theorem 3.1 .

TheOrem 3.5. Suppose that $G$ contains a connected nonabelian compact subgroup $K$. Then there is a compact subgroup $K^{*}$ of $G \times G$ such that $\mathrm{A}\left(G \times G / K^{*}\right)$ is not operator weakly amenable.

Proof. There exists a closed normal subgroup $N$ of $K$ such that $K / N$ is a compact Lie group [14, (28.61)(c)]. Moreover, we can, and shall, arrange for $K / N$ to be nonabelian. Indeed, if $s t \neq t s$ in $K$, find a neighbourhood $U$ of $e$ in $K$ such that $t s \notin s t U$; find in $U$ a compact subgroup $N$ so $K / N$ is a Lie group. Then by Theorem 3.1. $\mathrm{A}\left(K / N \times K / N: \Delta_{K / N}\right)$ is not operator weakly amenable. Let $q: K \times K \rightarrow K / N \times K / N$ be the quotient map and $K^{*}=q^{-1}\left(\Delta_{K / N}\right)$. Then by Lemma 3.4. $\mathrm{A}\left(K \times K: K^{*}\right) \cong \mathrm{A}(K / N \times$ $\left.K / N: \Delta_{K / N}\right)$ is not operator weakly amenable. Moreover, by Lemma 3.3 $\mathrm{A}\left(G \times G / K^{*}\right) \cong \mathrm{A}\left(G \times G: K^{*}\right)$ admits $\mathrm{A}\left(K \times K: K^{*}\right)$ as a quotient, and hence is not operator weakly amenable either.

3.3. Examples of hyper-Tauberian $\mathrm{A}(G / K)$. In this section we will always let $G$ denote a locally compact group. We shall generalise the fact that $\mathrm{A}(G)$ is hyper-Tauberian when the connected component of the identity is abelian [28, Thm. 21]. Our approach is inspired by that of [8, Thm. 3.3]. However, in dealing with coset spaces some extra technicalities arise. The following lemma deals with some of these technicalities.

Lemma 3.6. Suppose $G$ contains an open subgroup $G_{0}$ and a compact subgroup $K$ for which $\mathrm{A}\left(G_{0} / G_{0} \cap K\right)$ is hyper-Tauberian. Then $\mathrm{A}(G / K)$ is hyper-Tauberian.

Proof. We will identify $\mathrm{A}(G / K) \cong \mathrm{A}(G: K)$, etc., so we may work within the algebra $\mathrm{A}(G)$.

Let $H=G_{0} \cap K$. We will first establish that $\mathrm{A}\left(G_{0}: H\right)$ is boundedly isomorphic to a certain subalgebra of $\mathrm{A}(G: K)$.

Since $H$ is open in $K$, it is of finite index. Thus there is a finite set $\left\{k_{1}, \ldots, k_{n}\right\} \subset K$ for which

$$
K=\bigcup_{i=1}^{n} H k_{i} \quad \text { and } \quad H k_{i} \cap H k_{j}=\emptyset \quad \text { if } i \neq j .
$$

It then follows easily that

$$
G_{0} K=\bigcup_{i=1}^{n} G_{0} k_{i} \quad \text { and } \quad G_{0} k_{i} \cap G_{0} k_{j}=\emptyset \quad \text { if } i \neq j
$$

and thus $G K$ is a union of open cosets. Then the indicator function $1_{G K}$ is in $\mathrm{B}(G)$, and it is clear that $k \cdot 1_{G_{0} K}=1_{G_{0} K}$ for each $k \in K$. Hence $1_{G_{0} K} \mathrm{~A}(G: K)$ is a closed subalgebra of $\mathrm{A}(G: K)$. 
Now if $u \in \mathrm{A}(G: K)$, then $\left.u\right|_{G_{0}} \in \mathrm{A}\left(G_{0}: H\right)$ by Lemma 3.3. The restriction map

$$
\left.u \mapsto u\right|_{G_{0}}: 1_{G_{0} K} \mathrm{~A}(G: K) \rightarrow \mathrm{A}\left(G_{0}: H\right)
$$

is injective, for if $u$ in $\mathrm{A}(G: K)$ satisfies $u(s) \neq 0$ for some $s=s_{0} k$ in $G_{0} K$, where $s_{0} \in G_{0}$ and $k \in K$, then $u\left(s_{0}\right)=u(s) \neq 0$. Let us see that the map in (3.3) is surjective. Indeed, if $v \in \mathrm{A}\left(G_{0}: H\right)$ we define $\tilde{v}, w$ in $\mathrm{A}(G)$ by

$$
\tilde{v}(s)=\left\{\begin{array}{ll}
v(s) & \text { if } s \in G_{0} \\
0 & \text { otherwise }
\end{array} \quad \text { and } \quad w=\sum_{i=1}^{n} k_{i}^{-1} \cdot \tilde{v} .\right.
$$

Then $w \in 1_{G_{0} K} \mathrm{~A}(G)$ with $\left.w\right|_{G_{0}}=v$. Now, if $s_{0} \in G_{0}, h \in H$ and $k_{i}$ is as above, then, since $s_{0} h \in G_{0}$ and $v \in \mathrm{A}\left(G_{0}: H\right)$, we have

$$
w\left(s_{0} h k_{i}\right)=v\left(s_{0} h\right)=v\left(s_{0}\right)=w\left(s_{0}\right)
$$

and hence by (3.2), $w\left(s_{0} k\right)=w\left(s_{0}\right)$ for $s_{0}$ in $G_{0}$ and $k$ in $K$. Thus if $s \in G$ and $k \in K$, then either $s \in G_{0} K$ and we can find $s_{0}$ in $G_{0}$ and $k_{i}$ as above such that $s=s_{0} k_{i}$, so we have

$$
w(s k)=w\left(s_{0} k_{i} k\right)=w\left(s_{0}\right)=w\left(s_{0} k_{i}\right)=w(s) ;
$$

or $s \notin G_{0} K$, so for any $k$ in $K, s k \notin G_{0} K$ and thus $w(s k)=0=w(s)$. We conclude that $w \in \mathrm{A}(G: K)$. Thus the map in (3.3) is a contractive bijection which is also a homomorphism. It follows from the open mapping theorem that this map is an isomorphism. Thus, since $\mathrm{A}\left(G_{0}: H\right)$ is hyper-Tauberian, $1_{G_{0} K} \mathrm{~A}(G: K)$ is hyper-Tauberian.

Now let $T: \mathrm{A}(G: K) \rightarrow \mathrm{A}(G: K)^{*}$ be a bounded local operator, i.e. $N^{*} \circ T \circ N: \mathrm{A}(G / K) \rightarrow \mathrm{A}(G / K)^{*}$ is a local operator. We will show for $u_{1}, u_{2}, u_{3}$ in $\mathrm{A}(G: K)$, each having compact support, that

$$
\left\langle T\left(u_{1} u_{2}\right), u_{3}\right\rangle=\left\langle u_{1} T\left(u_{2}\right), u_{3}\right\rangle .
$$

Then it follows from Proposition 1.1(ii) that (3.4) holds for any $u_{1}, u_{2}, u_{3}$ in $\mathrm{A}(G: K)$.

Since $G_{0}$ is open, there are $t_{1}, \ldots, t_{n}$ in $G$ such that

$$
\bigcup_{j=1}^{3} \operatorname{supp}\left(u_{j}\right) \subset \bigcup_{i=1}^{n} t_{i} G_{0} \subset \bigcup_{i=1}^{n} t_{i} G_{0} K .
$$

The map $u \mapsto t_{i} * u$ is an isometric isomorphism from $1_{G_{0} K} \mathrm{~A}(G: K)$ to $1_{t_{i} G_{0} K} \mathrm{~A}(G: K)$ for each $i$, so each $1_{t_{i} G_{0} K} \mathrm{~A}(G: K)$ is hyper-Tauberian. Now let

$$
w_{1}=1_{t_{1} G_{0} K}, \quad w_{i}=1_{t_{i} G_{0} K}\left(1-\sum_{k=1}^{i-1} w_{k}\right) \quad \text { for } i=2, \ldots, n .
$$

Then each $w_{i}$ is an idempotent in $\mathrm{B}(G)$ with $k \cdot w_{i}=w_{i}$ for each $k$ in $K$. Moreover, $w_{i} w_{j}=0$ if $i \neq j$. For each $i$ the map $u \mapsto w_{i} u$ 
from $1_{t_{i} G_{0} K} \mathrm{~A}(G: K)$ to $w_{i} \mathrm{~A}(G: K)$ is a surjective homomorphism, so $w_{i} \mathrm{~A}(G: K)$ is hyper-Tauberian by [28, Thm. 12]. Then the algebra

$$
\mathcal{A}=\sum_{i=1}^{n} w_{i} \mathrm{~A}(G: K) \cong \bigoplus_{i=1}^{n} w_{i} \mathrm{~A}(G: K)
$$

is hyper-Tauberian, by [28, Cor. 13], and contains each $u_{j}$. The inclusion map $\iota: \mathcal{A} \rightarrow \mathrm{A}(G: K)$ is an $\mathcal{A}$-module map, so $\iota^{*} \circ T \circ \iota: \mathcal{A} \rightarrow \mathcal{A}^{*}$ is an $\mathcal{A}$-local operator. Hence $\iota^{*} \circ T \circ \iota$ is an $\mathcal{A}$-module map and (3.4) holds.

THEOREM 3.7. If $G$ has abelian connected component of the identity, $G_{e}$, then for any compact subgroup $K, \mathrm{~A}(G / K)$ is hyper-Tauberian.

Proof. As in the proof of the lemma above, we will identify $\mathrm{A}(G / K) \cong$ $\mathrm{A}(G: K)$, etc.

We will first assume that $G$ is almost connected.

Let $U$ be a neighbourhood of the identity in $G$. Then, by [20], there is a compact normal subgroup $N_{U} \subset U$ such that $\tilde{G}=G / N_{U}$ is a Lie group. Let $q: G \rightarrow \tilde{G}$ be the canonical quotient map and $\tilde{K}=q(K)$, so $\tilde{K}$ is a compact subgroup of $\tilde{G}$. The connected component of the identity of $\tilde{G}$ satisfies $\tilde{G}_{e}=\overline{q\left(G_{e}\right)}$ by [15, (7.12)]. Thus $\tilde{G}_{e}$ is abelian, and open in $\tilde{G}$ since the latter is a Lie group. It then follows that $\tilde{G}_{e} \cap \tilde{K}$ is normal in $\tilde{G}_{e}$ and $\mathrm{A}\left(\tilde{G}_{e}: \tilde{G}_{e} \cap \tilde{K}\right) \cong \mathrm{A}\left(\tilde{G}_{e} / \tilde{G}_{e} \cap \tilde{K}\right)$ is hyper-Tauberian by [28, Prop. 18]. Then it follows from Lemma 3.6 that $\mathrm{A}(\tilde{G}: \tilde{K})$ is hyper-Tauberian.

We let $K_{U}=q^{-1}(\bar{K})=K N_{U}$. Then Lemma 3.4 tells us that $\mathrm{A}\left(G: K_{U}\right)$ is isometrically isomorphic to $\mathrm{A}(\tilde{G}: \tilde{K})$, and hence is hyper-Tauberian. Since $K_{U} \supset K$, it follows that $\mathrm{A}\left(G: K_{U}\right) \subset \mathrm{A}(G: K)$.

Let $u \in \mathrm{A}(G: K)$, and $\varepsilon>0$. Fix a compact neighbourhood $V$ of $e$. Find a neighbourhood $U$ of $e$ such that $U \subset V$ and

$$
\|s \cdot u-u\|_{\mathrm{A}}<\frac{\varepsilon}{m(K V)} \quad \text { for } s \in U .
$$

Then find a normal subgroup $N_{U} \subset U$ as above and let

$$
P_{U} u=\int_{K_{U}} k \cdot u d k \in \mathrm{A}\left(G: K_{U}\right) .
$$

We note that for any $k=k^{\prime} n \in K N_{U}$ with $k^{\prime}$ in $K$ and $n$ in $N_{U}$ we have

$$
\begin{aligned}
\|k \cdot u-u\|_{\mathrm{A}} & =\left\|k^{\prime} \cdot(n \cdot u)-u\right\|_{\mathrm{A}}=\left\|n \cdot u-k^{\prime-1} \cdot u\right\|_{\mathrm{A}} \\
& =\|n \cdot u-u\|_{\mathrm{A}}<\frac{\varepsilon}{m(K V)}
\end{aligned}
$$

since $u \in \mathrm{A}(G: K)$ and right translation on $\mathrm{A}(G)$ is an isometry. Hence we find that

$$
\left\|P_{U} u-u\right\|_{\mathrm{A}} \leq \int_{K_{U}}\|k \cdot u-u\|_{\mathrm{A}} d k<\frac{\varepsilon}{m(K V)} m\left(K_{U}\right) \leq \varepsilon .
$$

Thus $\lim _{U \backslash\{e\}} P_{U} u=u$. 
Now let $T: \mathrm{A}(G: K) \rightarrow \mathrm{A}(G: K)^{*}$ be a bounded local operator. Since the inclusion $\iota: \mathrm{A}\left(G: K_{U}\right) \rightarrow \mathrm{A}(G: K)$ is an $\mathrm{A}\left(G: K_{U}\right)$-module map, and $\mathrm{A}\left(G: K_{U}\right)$ is hyper-Tauberian, $\iota^{*} \circ T \circ \iota: \mathrm{A}\left(G: K_{U}\right) \rightarrow \mathrm{A}\left(G: K_{U}\right)^{*}$ is an $\mathrm{A}\left(G: K_{U}\right)$-local map and hence an $\mathrm{A}\left(G: K_{U}\right)$-module map. Hence if $u_{1}, u_{2}, u_{3} \in \mathrm{A}(G: K)$, then

$$
\left\langle T\left(P_{U} u_{1} P_{U} u_{2}\right)-P_{U} u_{1} T\left(P_{U} u_{2}\right), P_{U} u_{3}\right\rangle=0 .
$$

Taking $U \searrow\{e\}$, as above, we obtain

$$
\left\langle T\left(u_{1} u_{2}\right)-u_{1} T\left(u_{2}\right), u_{3}\right\rangle=0 .
$$

and hence $T$ is an $\mathrm{A}(G: K)$-module map too.

Finally, if $G$ is not almost connected, we can find an almost connected open subgroup $G_{0}$. Then, from above, $\mathrm{A}\left(G_{0}: G_{0} \cap K\right)$ is hyper-Tauberian. We then appeal to Lemma 3.6.

We say $G$ is a [SIN]-group if there is a neighbourhood basis at $e$ consisting of sets invariant under inner automorphisms. We obtain for such groups a partial converse of Theorem 3.7, which is similar to [8, Thm. 3.7].

Corollary 3.8. If $G$ is a [SIN]-group, then $\mathrm{A}\left(G \times G / K^{*}\right)$ is hyperTauberian for every compact subgroup $K^{*}$ of $G \times G$ if and only if $G_{e}$ is abelian.

Proof. Sufficiency is an obvious consequence of Theorem 3.7. To see necessity, we first note that $G_{e}$ is a [SIN]-group and the Freudenthal-Weil theorem (see [22, 12.4.28]) tells us that $G_{e} \cong V \times K$ where $V$ is a vector group and $K$ a connected compact group. If $K$ is nonabelian, we appeal to Theorem 3.5 to see that there exists a subgroup $K^{*}$ of $G \times G$ such that $\mathrm{A}\left(G \times G / K^{*}\right)$ is not operator weakly amenable, hence not weakly amenable and not hyper-Tauberian.

3.4. Operator amenability of $\mathrm{A}(G / K)$. We recall that $[\mathrm{MAP}]_{K^{-}}$ groups were defined in Section 1.3 . We note that $\mathcal{T}_{f}(G) \mathrm{A}(G) \subset \mathrm{A}(G)$, since $\mathcal{T}_{f}(G)$ is a subalgebra of the Fourier-Stieltjes algebra $\mathrm{B}(G)$.

THEOREM 3.9. Let $G$ be an amenable locally compact group and $K$ a compact subgroup so that $G$ is $[\mathrm{MAP}]_{K}$. Then the following are equivalent:

(i) $\mathrm{A}(G / K)$ is operator amenable;

(ii) $(K \times K) \Delta$ is in the closed coset ring of $G \times G$.

Moreover, if $G$ is compact, each of the above is equivalent to

(iii) $\mathrm{I}_{\mathrm{A}_{\Delta}(G)}(K)$ has a bounded approximate identity.

Proof. Since the map $u \mapsto \check{u}$ is an isomorphism on $\mathrm{A}(G \times G)$, we see that $\mathrm{I}_{\mathrm{A}(G \times G)}((K \times K) \Delta)$ has a b.a.i. if and only if $\mathrm{I}_{\mathrm{A}(G \times G)}(\Delta(K \times K))$ 
has a b.a.i. It follows from [7] that $\mathrm{I}_{\mathrm{A}(G \times G)}(\Delta(K \times K))$ has a b.a.i. if and only if $\Delta(K \times K)$ is in the closed coset ring of $G \times G$. An application of Corollary 1.5(i) tells us that $\mathrm{I}_{\mathrm{A}(G \times G)}(\Delta(K \times K))$ has a b.a.i. if and only if $\mathrm{I}_{\mathrm{A}(G \times G / K \times K)}\left(\Delta_{G / K}\right)$ has a b.a.i., where $\Delta_{G / K}=\{(s, s) K \times K: s \in G\}$. Under the identification

$$
\mathrm{A}(G / K) \widehat{\otimes} \mathrm{A}(G / K) \cong \mathrm{A}(G \times G / K \times K)
$$

provided by Proposition $1.2, \mathrm{I}_{\mathrm{A}(G \times G / K \times K)}\left(\Delta_{G / K}\right)$ corresponds to the kernel of the multiplication map $m: \mathrm{A}(G / K) \widehat{\otimes} \mathrm{A}(G / K) \rightarrow \mathrm{A}(G / K)$. Since $\mathrm{A}(G / K)$ has a bounded approximate identity, this kernel has a bounded approximate identity if and only if $\mathrm{A}(G / K)$ is operator amenable, by the completely bounded version of a splitting result from [18] (also see [2, 3.10]). This establishes the equivalence of (i) and (ii).

If $G$ is compact, then by Corollary $1.5(\mathrm{i}), \mathrm{I}_{\mathrm{A}_{\Delta}(G)}(K)$ has a b.a.i. if and only if $\mathrm{I}_{\mathrm{A}(G \times G)}\left(K^{*}\right)$ has a b.a.i., where

$$
K^{*}=(K \times\{e\}) \Delta=(K \times K) \Delta .
$$

Hence it again follows from [7] that (ii) and (iii) are equivalent.

The situation that $(K \times K) \Delta$ is in the coset ring of $G \times G$ seems rare. It does occur, for example, when $K$ contains a subgroup $N$, of finite index, which is normal in $G$. Thus it is only in such cases that we know $\mathrm{A}(G / K)$ is operator amenable.

However, we gain some situations in which $(K \times K) \Delta$ is a set of local synthesis for $\mathrm{A}(G \times G)$.

THEOREM 3.10. If $G$ has abelian connected component of the identity and $K$ is a compact subgroup of $G$ so that $G$ is $[\mathrm{MAP}]_{K}$, then

(i) $(K \times K) \Delta$ is a set of local synthesis for $\mathrm{A}(G \times G)$.

Moreover, if $G$ is compact then

(ii) $K$ is a set of spectral synthesis for $\mathrm{A}_{\Delta}(G)$.

Proof. (i) By Theorem 3.7, $\mathrm{A}(G / K)$ is hyper-Tauberian. Hence it is operator hyper-Tauberian. Thus, by [28, Thm. 6], $\Delta_{G / K}=\{(s, s) K \times K: s \in G\}$ is a set of local synthesis for $\mathrm{A}(G \times G / K \times K)$, since the latter is isomorphic to $\mathrm{A}(G / K) \widehat{\otimes} \mathrm{A}(G / K)$ by Proposition 1.2 . (We note that [28, Thm. 6] is proved for the projective tensor product of a hyper-Tauberian algebra with itself. However, an inspection of the proof, coupled with the formula representing an arbitrary element of the operator projective tensor product in [4, 10.2.1], shows that it holds for an operator hyper-Tauberian algebra with the operator projective tensor product.) Then it follows from Corollary $1.5\left(\right.$ ii $\left.^{\prime}\right)$ that $\Delta_{G / K}^{*}=\Delta(K \times K)$ is a set of local synthesis for $\mathrm{A}(G \times G)$. 
Since $u \mapsto \check{u}$ is an isomorphism on $\mathrm{A}(G \times G),(K \times K) \Delta$ is also a set of local synthesis.

(ii) By Corollary 1.5(ii), $K$ is spectral for $\mathrm{A}_{\Delta}(G) \cong \mathrm{A}(G \times G / \Delta)$ if and only if $K^{*}=(K \times K) \Delta$ (see (3.5) ) is spectral for $\mathrm{A}(G \times G)$. We appeal to (i).

\section{Convolution}

4.1. Convolution on compact groups. We close this article by addressing, in part, the case of what happens if we replace the map $\Gamma$, in Section 1.2, with convolution.

We let $\mathcal{A}(G \times G)$ be as in Section 1.2 , and insist further that the group action of left translation is isometric on $\mathcal{A}(G \times G)$ and continuous on $G \times G$. We then define a group action $(r, f) \mapsto r \diamond f: G \times \mathcal{A}(G \times G) \rightarrow \mathcal{A}(G \times G)$ by

$$
r \diamond w(s, t)=w\left(s r, r^{-1} t\right)=(r, e) \cdot[(e, r) * w](s, t)=(e, r) *[(r, e) \cdot w](s, t) .
$$

We let $\check{\Delta}=\left\{\left(t, t^{-1}\right): t \in G\right\}$ and define

$$
\mathcal{A}(G \times G: \check{\Delta})=\{f \in \mathcal{A}(G \times G): r \diamond f=f \text { for every } r \text { in } G\} .
$$

We note that $w(s, t)=w\left(s_{1}, t_{1}\right)$ for $w \in \mathcal{A}(G \times G: \check{\Delta})$, provided $\left(s s_{1}^{-1}, t t_{1}^{-1}\right)$ $\in \check{\Delta}$ for $w \in \mathcal{A}(G \times G: \check{\Delta})$, even though $\check{\Delta}$ is not a subgroup unless $G$ is abelian. We then define

$$
\check{P}: \mathcal{A}(G \times G) \rightarrow \mathcal{A}(G \times G), \quad \check{P} w=\int_{G} r \diamond w d r,
$$

and

$$
\check{M}: \mathcal{A}(G \times G: \check{\Delta}) \rightarrow \mathcal{C}(G), \quad \check{M} f(s)=f(s, e) .
$$

Then $\check{P}$ is a contractive idempotent whose range is $\mathcal{A}(G \times G: \check{\Delta})$, in particular $\check{P}$ is a quotient map. The map $\check{M}$ is injective; we denote its range by $\mathcal{A}_{\check{\Delta}}(G)$ and assign to it the norm which makes $\check{M}$ an isometry. Then $\check{M}$ has inverse

$$
\check{N}: \mathcal{A}_{\check{\Delta}}(G) \rightarrow \mathcal{A}(G \times G: \check{\Delta}), \quad \check{N} f(s, t)=f(s t) .
$$

Finally, we define

$$
\check{\Gamma}: \mathcal{A}(G \times G) \rightarrow \mathcal{A}_{\check{\Delta}}(G), \quad \check{\Gamma}=\check{M} \circ \check{P} .
$$

If $\mathcal{A}(G \times G)$ contains an elementary product $f \times g$, then $\check{\Gamma} f \times g=f * g$. Thus, $\check{\Gamma}$ may be regarded simply as the convolution map.

4.2. Convolution on the Fourier algebras. We will now consider only the case where $\mathcal{A}(G \times G)=\mathrm{A}(G \times G)$. As in Section 1.2, it is easy to verify that $\check{\Gamma}: \mathrm{A}(G \times G) \rightarrow \mathrm{A}_{\check{\Delta}}(G)$ is a complete quotient map and $\mathrm{A}_{\check{\Delta}}(G) \subset \mathrm{A}(G)$. 
We recall that $\mathrm{A}_{\gamma}(G)$ is the subalgebra of $\mathrm{A}(G)$ defined in Section 1.2 . Example (iii). For this algebra we have

$$
f \in \mathrm{A}_{\gamma}(G) \Leftrightarrow \sum_{\pi \in \widehat{G}} d_{\pi}^{2}\|\hat{f}(\pi)\|_{1}<\infty
$$

and the latter quantity is the norm $\|f\|_{\mathrm{A}_{\gamma}}$, by [17, Prop. 2.5].

Theorem 4.1. We have $\mathrm{A}_{\breve{\Delta}}(G)=\mathrm{A}_{\gamma}(G)$ isometrically. Moreover, $\mathrm{A}_{\check{\Delta}}(G)=\mathrm{A}(G)$ if and only if $G$ admits an abelian subgroup of finite index.

Proof. We have a computation similar to that in Lemma 2.1. For $f$ in $\mathcal{C}(G)$ and $\pi$ in $\widehat{G}$ we have

$$
\begin{aligned}
\widehat{\widehat{N} f}(\bar{\pi} \times \bar{\pi}) & =\int_{G} \int_{G} f(s t) \pi(s) \otimes \pi(t) d t=\iint_{G} f(s) \pi\left(s t^{-1}\right) \otimes \pi(t) d t \\
& =\left[\int_{G} f(s) \pi(s) \otimes \pi(e) d s\right] \circ\left[\int_{G} \pi\left(t^{-1}\right) \otimes \pi(t) d t\right] \\
& =\left[\hat{f}(\pi) \otimes I_{\mathcal{H}_{\pi}}\right] \circ \frac{1}{d_{\pi}} U
\end{aligned}
$$

where $U$ is a unitary, in fact a permutation matrix, as we shall see below. Indeed, identify the linear operators on $\mathcal{H}_{\pi}$ with the matrix space $M_{d_{\pi}}$ via an orthonormal basis, and then identify $M_{d_{\pi}} \otimes M_{d_{\pi}} \cong M_{d_{\pi}^{2}}$. We obtain, using (2.2), the $(i j, k l)$ th entry

$$
\left(\int_{G} \pi\left(t^{-1}\right) \otimes \pi(t) d t\right)_{i j, k l}=\int_{G} \pi_{i j}\left(t^{-1}\right) \pi_{k l}(t) d t=\int_{G} \overline{\pi_{j i}(t)} \pi_{k l}(t) d t=\frac{1}{d_{\pi}} \delta_{i l} \delta_{j k}
$$

where $\delta_{i l}$ and $\delta_{j k}$ are the Kronecker $\delta$-symbols.

Thus it follows that

$$
\|\widehat{\widehat{N} f}(\bar{\pi} \times \bar{\pi})\|_{1}=\frac{1}{d_{\pi}}\left\|\hat{f}(\pi) \otimes I_{\mathcal{H}_{\pi}}\right\|_{1}=\|\hat{f}(\pi)\|_{1} .
$$

If $\pi \neq \sigma$ in $\widehat{G}$ then it can be shown, just as above, that $\widehat{\bar{N} f}(\pi \times \sigma)=0$.

We then obtain

$$
f \in \operatorname{ran} \check{\Gamma} \Leftrightarrow \sum_{(\pi, \sigma) \in \widehat{G \times G}} d_{\pi} d_{\sigma}\|\widehat{N} f(\pi \times \sigma)\|_{1}=\sum_{\pi \in \widehat{G}} d_{\pi}^{2}\|\hat{f}\|_{1}<\infty .
$$

This is precisely the characterisation obtained for $\mathrm{A}_{\gamma}(G)$ in (4.1).

It can be shown, as in Corollary 2.4, that $\mathrm{A}_{\gamma}(G)=\mathrm{A}(G)$ if and only if $G$ has an abelian subgroup of finite index.

Note that it follows from (2.7) and Theorems 2.2 and 2.6 that there are contractive inclusions

$$
\mathrm{A}_{\Delta^{2}}(G) \subset \mathrm{A}_{\gamma}(G) \subset \mathrm{A}_{\Delta}(G) .
$$


Also, note that since $u \mapsto \check{u}$ is an isometric isomorphism on $\mathrm{A}(G)$, the definition of $\mathrm{A}_{\gamma}(G)$ given in Section 1.2 , Example (iii), yields the equality

$$
\check{\Gamma}(\mathrm{A}(G) \widehat{\otimes} \mathrm{A}(G))=\check{\Gamma}\left(\mathrm{A}(G) \otimes^{\gamma} \mathrm{A}(G)\right) .
$$

Acknowledgments. Research of the first named author was supported by NSERC Grant 90749-00.

Research of the second named author was supported by an NSERC Post Doctoral Fellowship while at the University of Waterloo.

Research of the third named author was supported by NSERC Grant 312515-05.

\section{References}

[1] W. G. Bade, P. C. Curtis, and H. G. Dales, Amenability and weak amenability for Beurling and Lipschitz algebras, Proc. London Math. Soc. (3) 55 (1987), 359-377.

[2] P. C. Curtis and R. J. Loy, The structure of amenable Banach algebras, J. London Math. Soc. 40 (1989), 89-104.

[3] E. G. Effros and Z.-J. Ruan, On approximation properties for operator spaces, Int. J. Math. 1 (1990), 163-187.

[4] -, -, Operator Spaces, London Math. Soc. Monogr. 23, Clarendon Press, New York, 2000.

[5] P. Eymard, L'algèbre de Fourier d'un groupe localement compact, Bull. Soc. Math. France 92 (1964), 181-236.

[6] B. E. Forrest, Fourier analysis on coset spaces, Rocky Mountain J. Math. 28 (1998), 173-190.

[7] B. E. Forrest, E. Kaniuth, A. T. Lau, and N. Spronk, Ideals with bounded approximate identities in Fourier algebras, J. Funct. Anal. 203 (2003), 286-304.

[8] B. E. Forrest and V. Runde, Amenability and weak amenability of the Fourier algebra, Math. Z. 250 (2005), 731-744.

[9] B. E. Forrest, E. Samei, and N. Spronk, Weak amenability of Fourier algebras on compact groups, Indiana Univ. Math. J. 58 (2009), 1379-1394.

[10] B. E. Forrest and P. J. Wood, Cohomology and the operator space structure of the Fourier algebra and its second dual, ibid. 50 (2001), 1217-1240.

[11] F. Ghahramani and R. Stokke, Approximate amenability and pseudo-amenability of the Fourier algebra, ibid. 56 (2007), 909-930.

[12] N. Groenbaek, A characterization of weakly amenable Banach algebras, Studia Math. 94 (1989), 149-162.

[13] C. S. Herz, Harmonic synthesis for subgroups, Ann. Inst. Fourier (Grenoble) 23 (1973), 91-123.

[14] E. Hewitt and K. A. Ross, Abstract Harmonic Analysis II, Grundlehren Math. Wiss. 152, Springer, New York, 1970.

[15] -, -, Abstract Harmonic Analysis I, 2nd ed., Grundlehren Math. Wiss. 115, Springer, New York, 1979.

[16] B. E. Johnson, Cohomology in Banach algebras, Mem. Amer. Math. Soc. 127 (1972).

[17] - Non-amenability of the Fourier algebra of a compact group, J. London Math. Soc. 50 (1994), 361-374. 
[18] A. Ya. Khelemskiı̌, Flat Banach modules and amenable algebras, Trudy Moskov. Mat. Obshch. 47 (1982), 179-218 (in Russian); English transl.: Trans. Moscow Math. Soc. 1985, 199-244.

[19] V. Losert, On tensor products of Fourier algebras, Arch. Math. (Basel) 43 (1984), 370-372.

[20] D. Montgomery and L. Zippin, Topological Transformation Groups, 4th ed., Interscience Tracts Pure and Appl. Math. 1, Wiley, New York, 1966.

[21] C. C. Moore, Groups with finite dimensional irreducible representations, Trans. Amer. Math. Soc. 166 (1972), 401-410.

[22] T. W. Palmer, Banach Algebras and the General Theory of *-algebras. Volume II. *-algebras, Encyclopedia Math. Appl. 79, Cambridge Univ. Press, Cambridge, 2001.

[23] B. D. Park and E. Samei, Smooth and weak synthesis of the anti-diagonal in Fourier algebras of Lie groups, J. Lie Theory, accepted; arXiv:0809.2806v1.

[24] R. J. Plymen, Fourier algebra of a compact Lie group, arXiv:math.FA/0104018.

[25] Z.-J. Ruan, The operator amenability of $A(G)$, Amer. J. Math. 117 (1995), 14491474.

[26] V. Runde, Lectures on Amenability, Lecture Notes in Math. 1774, Springer, Berlin, 2002 .

[27] E. Samei, Bounded and completely bounded local derivations from certain commutative semisimple Banach algebras, Proc. Amer. Math. Soc. 133 (2005), 229-238.

[28] - Hyper-Tauberian algebras and weak amenability of Figà-Talamanca-Herz algebras, J. Funct. Anal. 231 (2006), 195-220.

[29] N. Spronk, Operator weak amenability of the Fourier algebra, Proc. Amer. Math. Soc. 130 (2002), 3609-3617.

[30] -, Operator space structure on Feichtinger's Segal algebra, J. Funct. Anal. 248 (2007), 152-174.

[31] N. Spronk and L. Turowska, Spectral synthesis and operator synthesis for compact groups, J. London Math. Soc. (2) 66 (2002), 361-376.

[32] M. Takesaki and N. Tatsuuma, Duality and subgroups, II, J. Funct. Anal. 11 (1972), 184-190.

[33] N. Th. Varopoulos, Tensor algebras and harmonic analysis, Acta Math. 119 (1967), $51-112$.

Brian E. Forrest, Nico Spronk

Department of Pure Mathematics

University of Waterloo

Waterloo, ON N2L 3G1, Canada

E-mail: beforres@uwaterloo.ca nspronk@uwaterloo.ca
Ebrahim Samei

Department of Mathematics \& Statistics University of Saskatchewan Saskatoon, SK S7N 5E6, Canada E-mail: samei@math.usask.ca 